Portland State University

PDXScholar

Civil and Environmental Engineering Master's

Project Reports

2014

\title{
A Comparative Assessment of Crowded Source Travel Time Estimates: A Case Study of Bluetooth vs INRIX on a Suburban Arterial
}

Fahad Fasha Alhajri

Portland State University

Follow this and additional works at: https://pdxscholar.library.pdx.edu/cengin_gradprojects

Part of the Civil Engineering Commons, and the Transportation Engineering Commons Let us know how access to this document benefits you.

\section{Recommended Citation}

Alhajri, Fahad Fasha, "A Comparative Assessment of Crowded Source Travel Time Estimates: A Case Study of Bluetooth vs INRIX on a Suburban Arterial" (2014). Civil and Environmental Engineering Master's Project Reports. 4.

https://doi.org/10.15760/CEEMP.25

This Project is brought to you for free and open access. It has been accepted for inclusion in Civil and Environmental Engineering Master's Project Reports by an authorized administrator of PDXScholar. Please contact us if we can make this document more accessible: pdxscholar@pdx.edu. 
A COMPARITIVE ASSESSMENT OF CROWDED SOURCE TRAVEL TIME ESTIMATES:

A CASE STUDY OF BLUETOOTH VS INRIX ON A SUBURBAN ARTERIAL

BY

FAHAD F A SH A ALHAJRI

A research project report submitted in partial fulfillment

of the requirement for the degree of

\author{
MASTER OF SCIENCE \\ IN \\ CIVIL AND ENVIRONMENTAL ENGINEERING
}

Project Advisor:

Christopher M. Monsere

Portland State University

(C)2014 


\section{ACKNOWLEDGMENTS}

I would like to gratefully acknowledge my wonderful advisor Dr. Christopher Monsere whose support and guidance enabled me to complete this project. It was an honor to work with someone who really cares about his students.

I would like to also extend my acknowledgement to Jon Makler, Steve Hansen, Joel Barnett at PSU who contributed to the data collection and analysis of an earlier evaluation on which this research is based on. Dr. Robert Bertini for coordinating PSU's access to the INRIX data after ODOTs approval and support. Dr. Robert Fountain and Alexander Bigazzi for their valuable input on time series analysis. Ted Trapanier at INRIX for providing helpful orientation on using the INRIX data for the analysis.

Finally, none of this would have been possible without the unconditional love, endless support and extraordinary encouragement of my parents, Fawaz and Ahlam, and my aunt Dr. Faiza. 


\section{ABSTRACT}

Travel time is one of the most widely used measures of traffic performance monitoring for the transportation systems. It is a simple concept that refers to the time required to traverse between two points of interest. Travel time is communicated and used by a wide variety of audience such as commuters, media reporters, and transportation engineers and planners. Recent developments within the wireless communication area made it possible to collect travel time data at a relatively low cost. These emerging technologies include mobile phone based technologies, in-vehicle navigation technologies and automatic vehicle identification technologies. Although these technologies offer a great collection source for travel time data, they have different levels of accuracy. In this research two sources of travel time data were evaluated. These sources of data were the INRIX travel time data and the Bluetooth travel time data. The granularity of the INRIX and the Bluetooth data were high in which travel time estimates were reported at ane minute interval. A total of 42 GPS vehicle probe surveys were carried out in three different days to evaluate the accuracy of the INRIX and the Bluetooth travel time estimates. Statistical measures such as the mean absolute error (MAE) and the mean absolute percent error (MAPE) were calculated for a total of 6 segments and 3 time periods (midday, pm peak, and weekend). The INRIX estimates during the midday were either within 0.36 minutes or $22 \%$ of the ground truth probe runs, while the Bluetooth estimates during the pm peak were either within 1 minute or $24 \%$ of the ground truth probe runs. In addition to hypothesis testing for 13,541 matched-pairs observation, correlation testing was carried out to evaluate the behavior of the Bluetooth and INRIX time series. 


\section{TABLE OF CONTENTS}

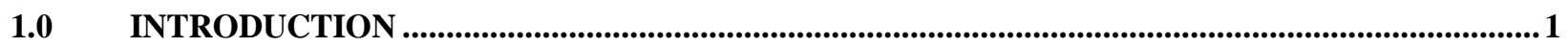

2.0 LITERATURE REVIEW

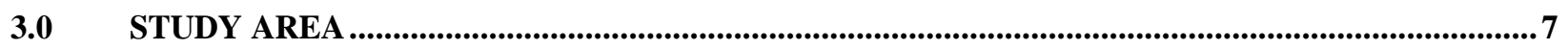

4.0 DATA .

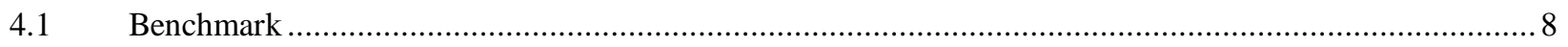

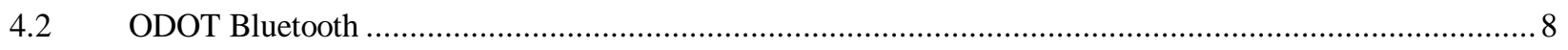

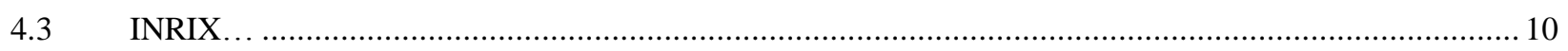

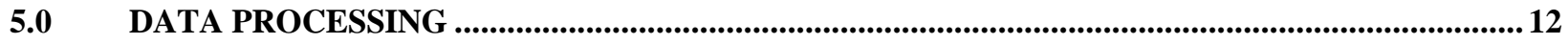

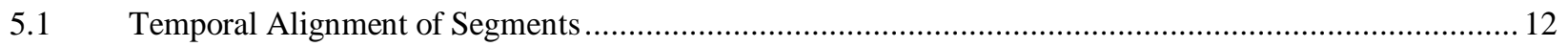

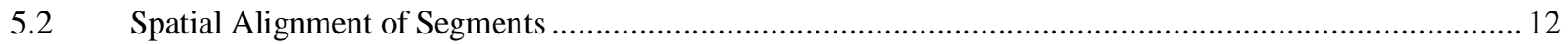

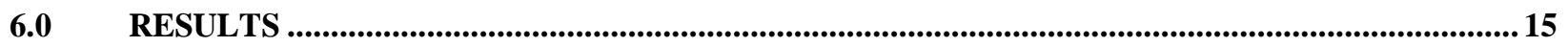

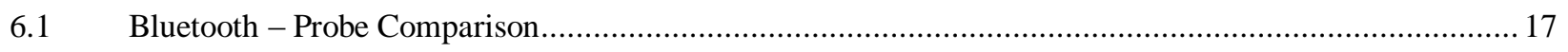

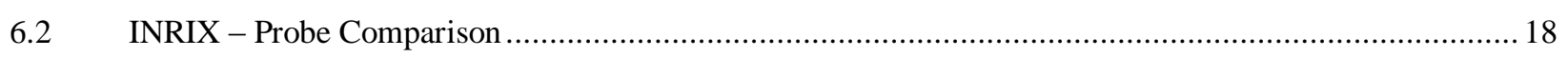

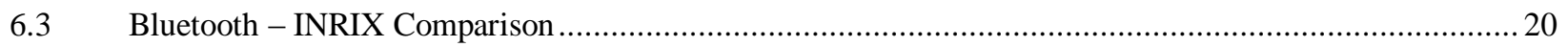

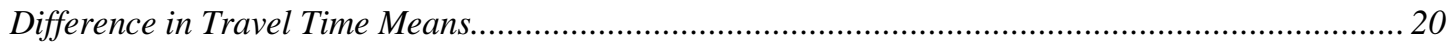

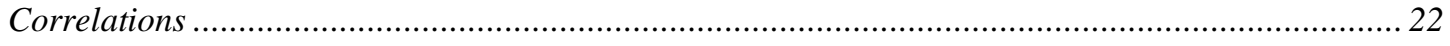

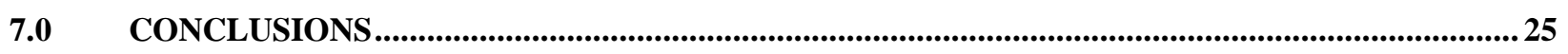

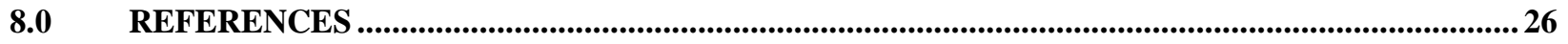

\section{APPENDICES}

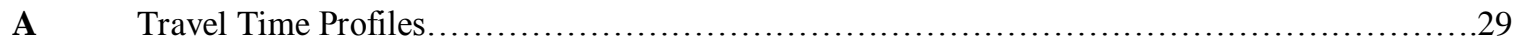

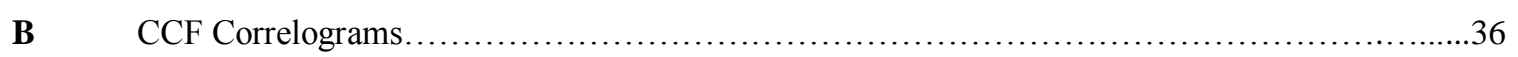




\section{LIST OF TABLES}

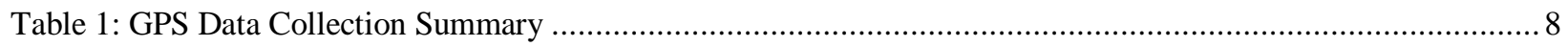

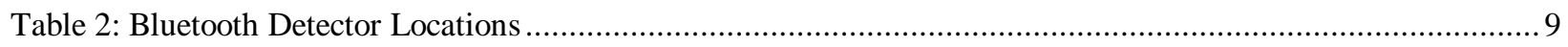

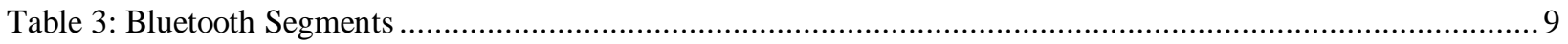

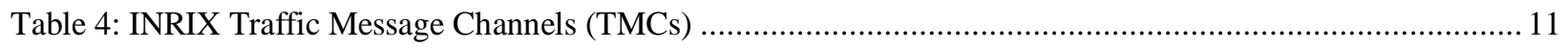

Table 5: Summary of Statistical Measures for (Bluetooth \& INRIX) to Probe ....................................................... 19

Table 6: Matched Pairs T-Test for the Difference in Means .............................................................................. 20

Table 7: P-Values for the Correlation Hypothesis testing (P-Value > 0.05 shaded $)$............................................ 23

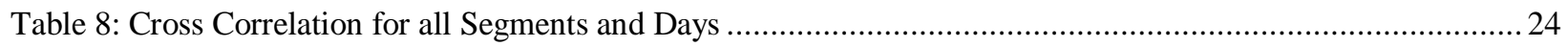




\section{LIST OF FIGURES}

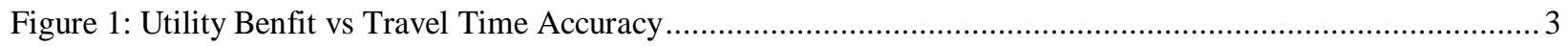

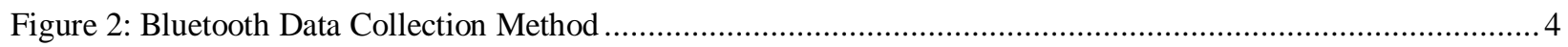

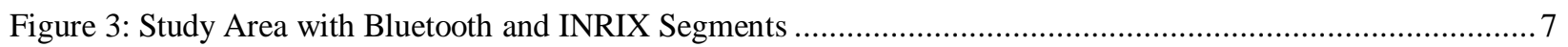

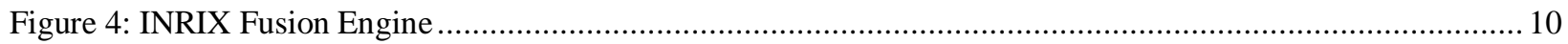

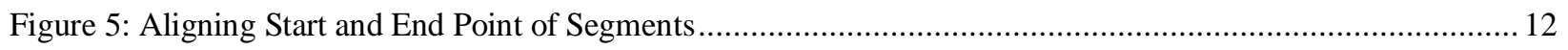

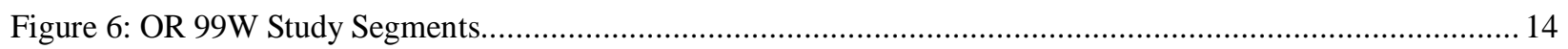

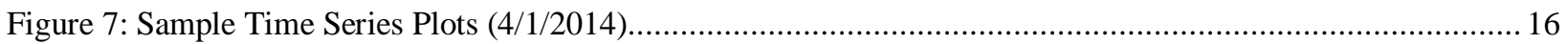

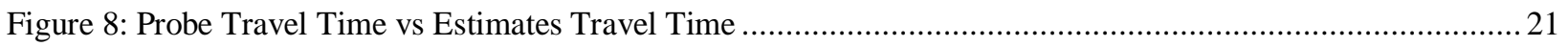

Figure 9: Time Series Profile of McDonald to Durham (4/1/2014) ...................................................................... 22

Figure 10: CCF Correlogram for McDonald to OR-217 (4/1/2014) ............................................................... 24

Figure 11: Travel Time Profiles for Southbound Segments (4/1/2014) ............................................................. 30

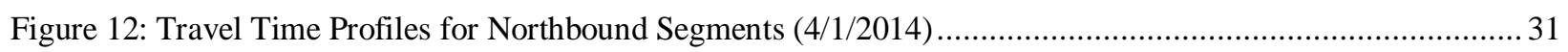

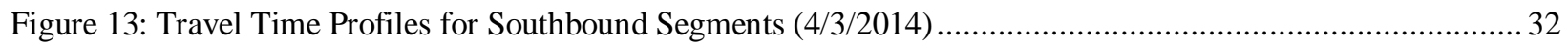

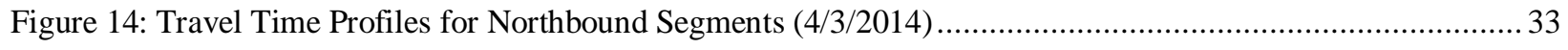

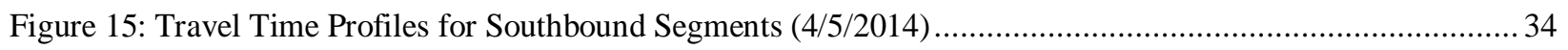

Figure 16: Travel Time Profiles for Northbound Segments $(4 / 5 / 2014)$............................................................ 35

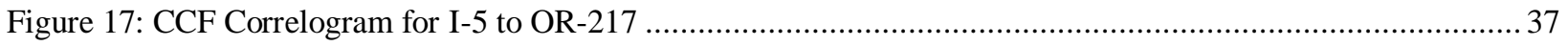

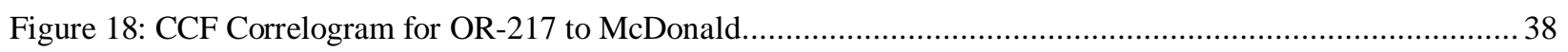

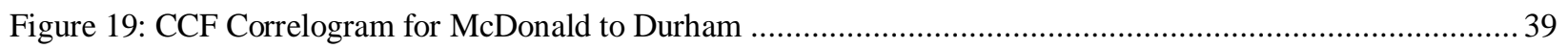

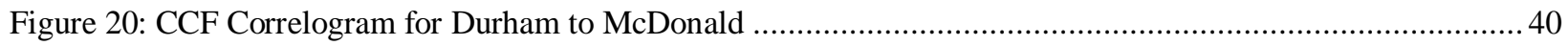

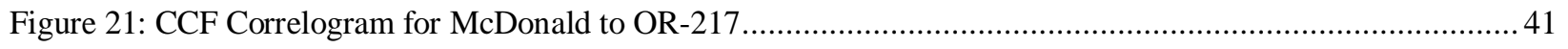

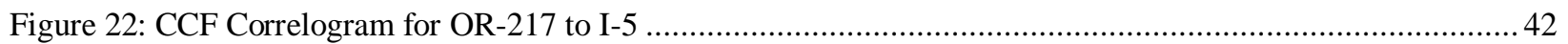




\subsection{INTRODUCTION}

Travel time is one of the most widely used measures of traffic performance monitoring for the transportation systems. It is a simple concept that refers to the time required to traverse between two points of interest. Travel time is communicated and is used by a wide variety of audience such as commuters, media reporters, and transportation engineers and planners. Commuters use travel time to locate their housing with respect to their work location. The media reports an expected delay in travel time along a freeway when an incident takes place. Engineers and planners use travel time to evaluate transportations facilities and quantify capital investment.

Traditionally, the level of service (LOS) as in the Highway Capacity Manual (HCM) and AASHTO Geometric Design of Highways and Streets measured the performance of a transportation facility. The LOS assigns letters "A" through "F" to a transportation facility, "A" as being best and "F" as being worst. During the development of the level of service, the availability of transportation data was very limited. Presently, the Intelligent Transportation Systems (ITS) deployments and the infiltration of new technologies into the market made it possible to immensely increase the availability of transportation data. These technologies fall into one of two categories the first being fixed-point technologies and the second being probe vehicle technologies (Tantiyanugukchai, 2004).

Fixed-point technologies (Inductive loop detectors, CCTV Cameras, and Automatic Vehicle Identification) collect various traffic characteristics of a stream at predetermined points where the sensors are installed. Probe vehicle technologies are vehicles infused into the traffic stream with a capability of recording position and time data (GPS receiver) while in the stream. The data is then downloaded and synthesized to obtain traffic measures such as travel time and travel speed of the transportation system (Izadpanah, 2010).

Recent developments within the wireless communication area made it possible to collect traffic data at a relatively low cost. These emerging technologies include mobile phone based technologies, in-vehicle navigation technologies and automatic vehicle identification

technologies. The mobile phone based technologies tracks the position of a mobile phone through cellular towers or GPS receivers impeded in the phones. The in-vehicle navigation technologies track the position of vehicles using GPS receivers, which are then transmitted to a 
server. The server receives the positions of the vehicle through either cellular network automatically or manually when the owner connects the navigation device to the Internet for updating purposes. The automatic vehicle identification (AVI) technologies covers a large spectrum of technologies such as radio frequency identification (RFID), automatic license plate recognition, Bluetooth ... etc. In all of the AVI technologies the vehicle is identified upstream at location " $\mathrm{A}$ " and the timestamp is recorded. The vehicle is then identified again downstream at location " $\mathrm{B}$ " and a timestamp is recorded. The difference between a timestamp recorded at location " $\mathrm{A}$ " and a timestamp recorded at location " $\mathrm{B}$ " is the travel time spent by the vehicle to travel between point "A" and "B" (Izadpanah and Hellinga, 2007).

Although previously mentioned technologies offer a great collection source for travel time data, they have different levels of accuracy. There have been very few side-by-side assessments and comparative analyses conducted for these technologies. As a result, the objective of this study was to compare the INRIX travel time data to the traditional Bluetooth travel time data. The granularity of the INRIX and the Bluetooth data were high in which travel time estimates were reported at a one minute interval. The study area for this research is approximately a 4.2-mile section of a suburban arterial (Oregon route 99W). GPS vehicle probe surveys were carried out in three different days to evaluate the accuracy of the INRIX and the Bluetooth travel time estimates. Statistical measures such as the mean absolute error (MAE) and the mean absolute percent error (MAPE) were calculated for a total of 6 segments and 3 time periods (midday, pm peak, and weekend). Hypothesis testing for 13,541 matched-pairs observation was conducted to determine whether or not the travel time collected by the Bluetooth method significantly differ from the INRIX method. Moreover, correlation testing was carried out to evaluate the behavior of the Bluetooth and INRIX time series.

This research is organized as follows: (1) Literature review provides a summary of the efforts in evaluating the travel time data; (2) Study area describes the location where the datasets were collected; (3) Data describes the datasets which were used in this research; (4) Data processing describes the methodology used in preparing the datasets; (5) Results present the outcome of the evaluation; (6) Conclusion summarizes the findings of this research. 


\subsection{LITERATURE REVIEW}

The reliability and accuracy of technologies that predicts travel time is important to road users. Toppen and Wunderlich (2003) studied the relationship between the error in travel time and the utility benefit to road users. The results of the study showed that when the accuracy drops below a certain threshold, the users are better off using their own travel experience than to use the travel time predicted by the Advanced Travelers Information Systems. The relationship depicting the utility gained by travelers with respect to the error in travel time estimation for the case study is shown in Figure 1. The $\mathrm{x}$-axis represent the percent error in travel time, while the y-axis represent the per trip utility in dollars. The curves represent the utility gained by the trip maker at four time periods: am peak in dark blue, pm peak in green, off peak in light blue, and all time period in red. For a 25 minute perfect trip ( $0 \%$ error), the trip maker was determined to realize a $\$ 2.00$ utility. From the figure, an error ranging between $13 \%$ and $21 \%$ results in a negative utility. In this research the acceptable threshold for the error was defined to be $25 \%$.

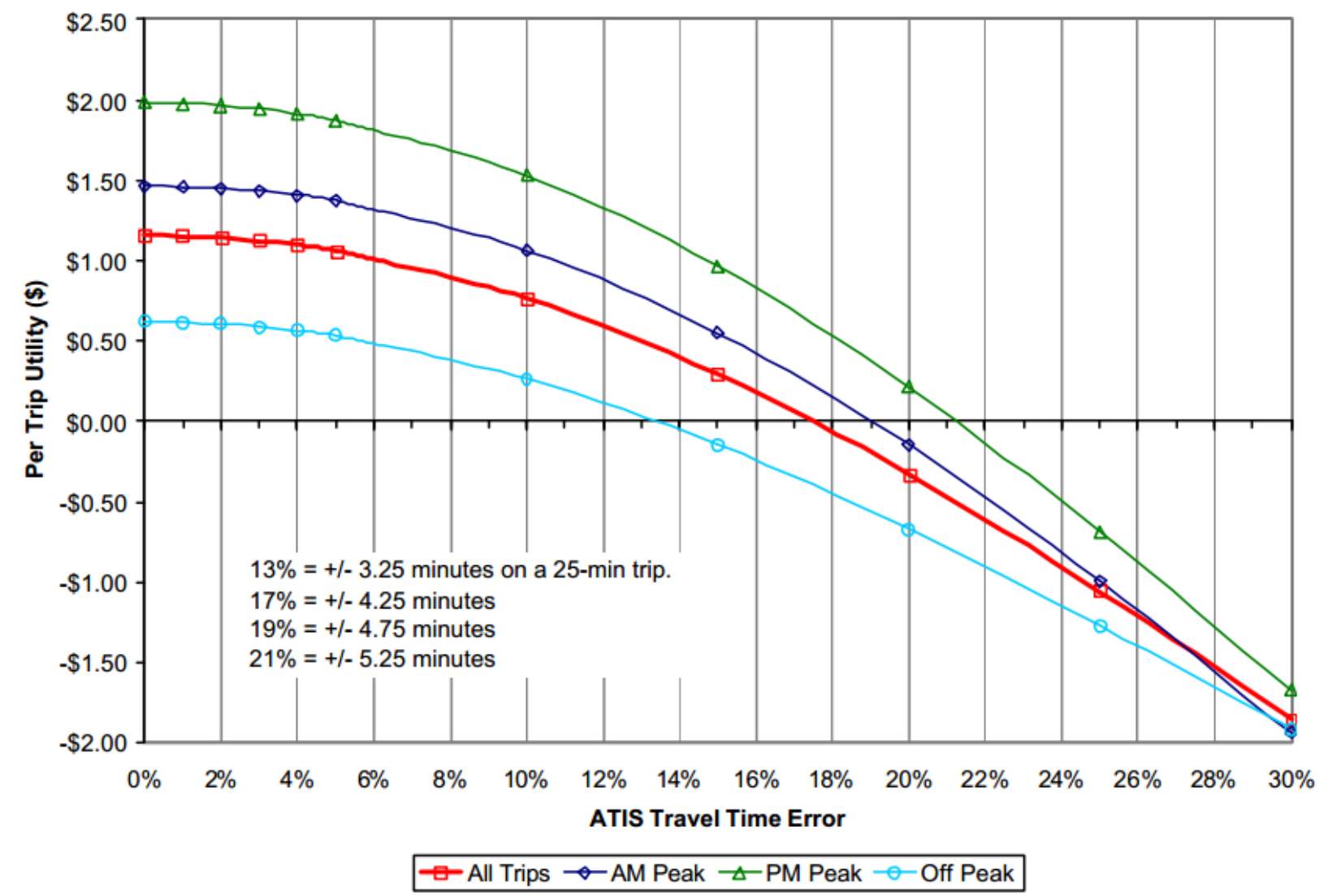

Figure 1: Utility Benfit vs Travel Time Accuracy

Source: Toppen and Wunderlich (2003) 
The Bluetooth technology has become the new source for travel time estimation due its cost effectiveness. Vehicles with electronic devices (cell phones, vehicle radios, PCs... etc.) that are equipped with Bluetooth technology emit waves that can be detected by Bluetooth receivers. These emissions are detected only when the device is set to discovery mode. Each device equipped with Bluetooth technology has a specific anonymous identifier called a Media Access Control (MAC) address. By placing two Bluetooth detectors, one at the beginning of the segment and one at the end of it, a timestamp is recorded when vehicles are entering the segment and when vehicles are leaving it. The matching of a specific MAC address associated with a vehicle at the entry and exit is used to calculate the travel time for that vehicle. An overview of the Bluetooth technology is presented in Figure 2. The accuracy and reliability of the Bluetooth technology for the purpose of travel time estimation is a topic discussed by several authors (Wasson et al. (2008), Qyale et al. (2010), Malinovskiy et al. (2010), Haghani et al. (2010), and Araghi et al. (2012)).

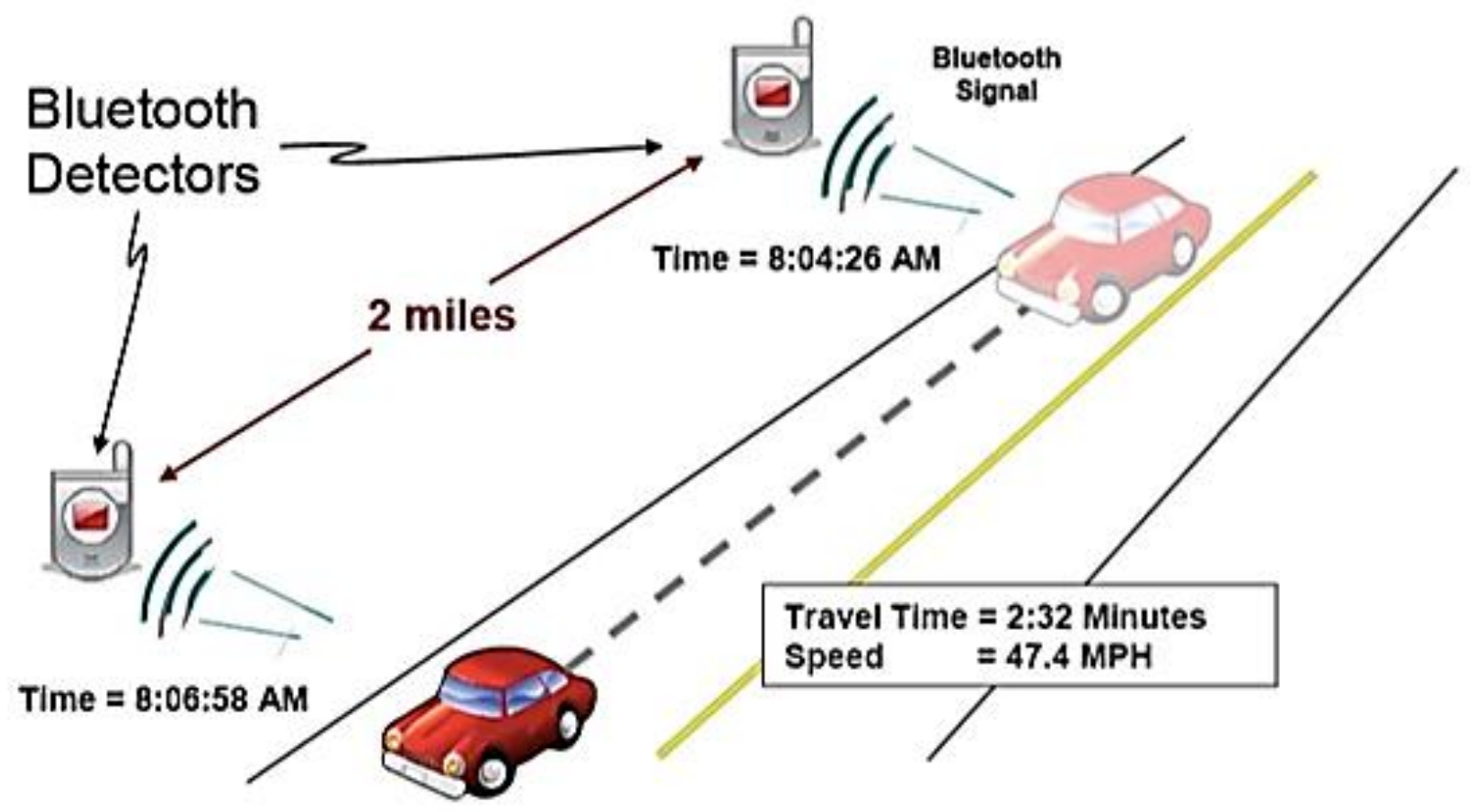

Figure 2: Bluetooth Data Collection Method Source: Haghani et al. (2009) 
Few studies were conducted to assess and evaluate the quality of vehicle probe data at a high level of detail. Haghani et al. (2009) from the University of Maryland validated the INRIX data for the I-95 corridor. The evaluation was carried out by comparing the INRIX data to ground truth data. Their evaluation followed three stages: (1) collect ground truth data, (2) establish the statistical measures for comparison of INRIX GPS data to ground truth, and (3) compare the data to ground truth and draw conclusions. In the process of collecting the ground truth data they ruled out the traditionally floating car method. They decided not to use the traditional floating car method because it would have been very costly to apply in their large network (1500 miles of freeway and 1000 miles of arterials). Instead of using the floating car method they used the Bluetooth technology.

Since the Bluetooth method of collecting travel time data is fairly a new method, Haghani et al. (2009) validated its accuracy by comparing it to ground truth data collected by GPS Probe vehicle runs. The comparison was performed by conducting a statistical hypothesis test to determine whether or not the means speeds collected by the Bluetooth method significantly differ from the mean speeds collected from floating car runs. A total of nine days of floating car testing was performed in the states of Maryland and North Virginia as a base to determine the accuracy of the Bluetooth data. The result of the statistical analysis showed the Bluetooth data to be a consistent and accurate for field measurement of travel times. The Bluetooth data was then used to evaluate the INRIX GPS data. For the purpose of the statistical analysis, the speed was broken down into 4 bins: 0 to $30 \mathrm{mph}, 30$ to $45 \mathrm{mph}, 45$ to $60 \mathrm{mph}$, and greater than $60 \mathrm{mph}$. Haghani et al. (2009) used the average absolute speed error (ASSE) and the speed error bias (SEB) as a statistical measures to compare the INRIX data to the Bluetooth data. They found that the INRIX data fell within the SEM band, the ASSE value to be less than $10 \mathrm{mph}$, and the SEB value to less than $5 \mathrm{mph}$. It was then concluded that the INRIX travel time and speed data to have a satisfying accuracy.

The Minnesota DOT compared the INRIX travel time data to loop detector data on an urban freeway. The INRIX travel time was found most accurate during peak periods and at speeds nearing the posted speed limit. The evaluation showed that $98 \%$ of the INRIX travel time estimates fell within 2 minutes or $20 \%$ of the loop detector travel times (MnDOT, 2012). Moreover, the Washington DOT conducted a study to evaluate the accuracy and reliability of 
several ATIS technologies on I-90 (rural freeway) and SR 522 (urban arterial). In the study, the automatic license plate reader (ALPR) system was used to evaluate the accuracy of the Bluetooth and INRIX travel time estimates. Measures such as Mean Absolute Deviation (MAD), Mean Absolute Error (MAE), and Mean Absolute Percent Error (MAPE) were calculated to assess the accuracy of the estimates. The results of the study showed the Bluetooth estimate to have a lower MAPE value than the INRIX estimates over the course of the day. The Bluetooth displayed lower accuracy during the night where sampling was really low. Futhermore, The Bluetooth and INRIX estimates were examined on a segment for a time period with a road closure. The Bluetooth continued on reporting the travel time for 30 minutes after road closure, while the INRIX failed to show a reaction to road closure. It was concluded from the study that the Bluetooth had a higher overall reliable travel time than the INRIX (WSDOT, 2014). 


\subsection{STUDY AREA}

The study area of this research is approximately a 4.2-mile section of the State Highway 99W corridor between the Durham Road/OR 99W intersection at the south end and the I-5 interchange at the north end. Highway $99 \mathrm{~W}$ is 5 lanes wide and at minimum 4 lane wide at some sections. The posted speed limit in the corridor is between 35 and $40 \mathrm{mph}$. The highway carries approximately 38,000 vehicles a day with $1.50 \%$ heavy vehicles (ODOT, 2013). The corridor is surrounded by a variety of land uses with the majority being retail and commercial services. A map showing the corridor (purple), the Bluetooth segment boundaries (blue) and the INRIX TMC boundaries (green) is presented in Figure 3

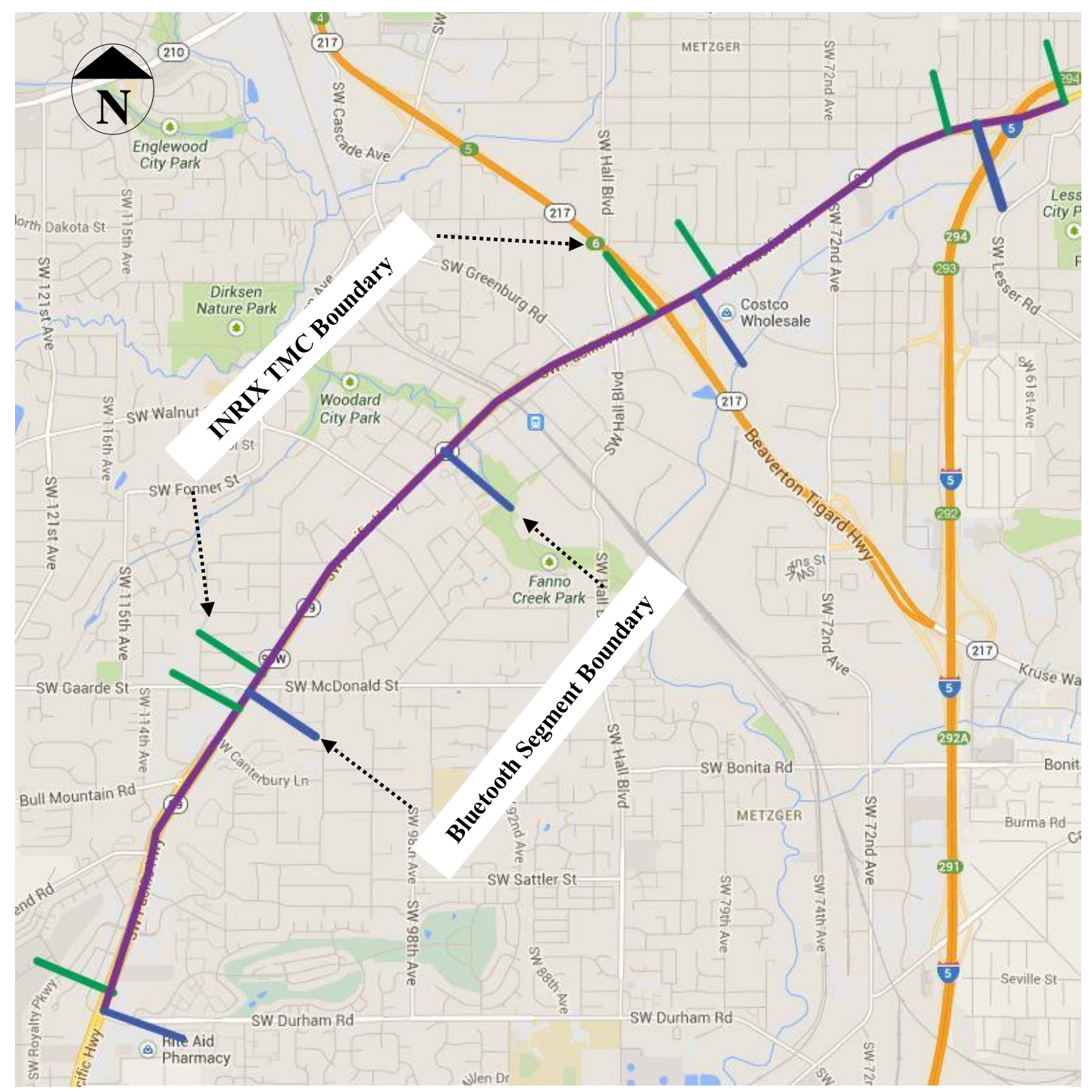

Figure 3: Study Area with Bluetooth and INRIX Segments 


\subsection{DATA}

In this study two data sources were used:

- $\quad$ Bluetooth, and

- INRIX

The GPS vehicle probes were used as a benchmark to evaluate the accuracy of these data sources. The GPS probe vehicle survey was conducted by the ITS lab at Portland State University. The Bluetooth travel time data was obtained from the Oregon Department of Transportation System and the INRIX Travel Time data was provided by INRIX under a license purchased by ODOT. The following subsection will describe each dataset.

\subsection{Benchmark}

In order to obtain ground truth data, a total of 42 GPS probe runs were carried out in three-time periods: midday, pm peak, and weekend. The data was collected on Tuesday 4/1/2014, Thursday 4/3/2014, and Saturday 4/5/2014. The GPS probe runs followed the floating car methodology outlined in the FHWA Travel Time Data Collection Handbook (FHWA, 1998). A detailed summary of the collection effort is presented in Table 1.

Table 1: GPS Data Collection Summary

\begin{tabular}{c|c|c|c|c|c|c}
\hline Date & Day of Week & Start & End & Hours & Time Period & Total Number of Runs \\
\hline $4 / 1 / 2014$ & Tuesday & $12: 00$ & $14: 00$ & 2 & Midday & 11 \\
\hline $4 / 1 / 2014$ & Tuesday & $16: 00$ & $18: 00$ & 2 & PM Peak & 9 \\
\hline $4 / 3 / 2014$ & Thursday & $12: 00$ & $14: 00$ & 2 & Midday & 4 \\
\hline $4 / 3 / 2014$ & Thursday & $16: 00$ & $18: 00$ & 2 & PM Peak & 8 \\
\hline $4 / 5 / 2014$ & Saturday & $11: 00$ & $13: 00$ & 2 & Weekend & 10 \\
\hline \multicolumn{7}{c}{ Total } \\
\hline
\end{tabular}

\subsection{ODOT Bluetooth}

The Bluetooth travel time data was retrieved from 5 Bluetooth detectors in the study area. Each Bluetooth detector records the MAC address and the timestamp associated with each travelling vehicles containing a Bluetooth device set to discovery mode. The location of these Bluetooth Detectors along Oregon route $99 \mathrm{~W}$ are presented in Table 2 
Table 2: Bluetooth Detector Locations

\begin{tabular}{c|l|c|c|c}
\hline Detector ID & \multicolumn{1}{|c|}{ Detector Location } & Mile Post & Latitude & Longitude \\
\hline 467 & $99 \mathrm{~W} / \mathrm{I}-5$ & 7.58 & $\mathrm{~N} 45.44326$ & $\mathrm{~W} 122.74279$ \\
\hline 468 & 99W / OR-217 & 8.55 & $\mathrm{~N} 45.43568$ & $\mathrm{~W} 122.75995$ \\
\hline 469 & 99W / Main & 9.46 & $\mathrm{~N} 45.42893$ & $\mathrm{~W} 122.77569$ \\
\hline 470 & 99W / McDonald & 10.39 & $\mathrm{~N} 45.41856$ & $\mathrm{~W} 122.78755$ \\
\hline 471 & 99W / Durham & 11.49 & $\mathrm{~N} \mathrm{45.40456}$ & $\mathrm{W} 122.79645$ \\
\hline
\end{tabular}

Once the MAC address is matched between two consecutive detectors, a travel time is calculated for the traversed segment between the detectors. The Oregon DOT system uses data collection devices designed by Kim and Porter (Porter and Kim, 2011). Since there are 5 detectors in the study area there are 4 segments in each direction. The Bluetooth travel time data that was obtained from Oregon DOT's own system contains attributes such as: a timestamp, a segment ID and an average travel time for that segment. The Bluetooth segments are summarized in Table 3.

Table 3: Bluetooth Segments

\begin{tabular}{|c|c|c|c|c|}
\hline Direction & Segment ID & Segment Begins & Segment Ends & Segment Length (miles) \\
\hline \multirow{4}{*}{ 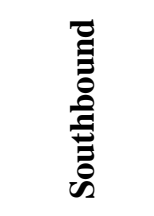 } & 2293 & $\mathrm{I}-5$ & OR-217 & 0.97 \\
\hline & 2295 & OR-217 & Main & 0.91 \\
\hline & 2297 & Main & McDonald & 0.93 \\
\hline & 2299 & McDonald & Durham & 1.10 \\
\hline \multirow{4}{*}{ 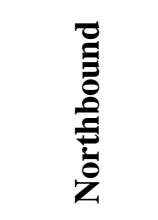 } & 2300 & Durham & McDonald & 1.10 \\
\hline & 2298 & McDonald & Main & 0.93 \\
\hline & 2296 & Main & OR-217 & 0.91 \\
\hline & 2294 & OR-217 & I-5 & 0.97 \\
\hline
\end{tabular}

The Bluetooth data was available at a high resolution and the travel time estimates were reported at a one-minute interval. For each minute interval, the travel time estimate is an average of all vehicles observed for that minute interval. Moreover, the data processing of the outliers to generate travel times is done by the Oregon DOT software. 


\subsection{INRIX}

INRIX is a private party that collects information about the roadway conditions. It accomplishes this mission with its smart drive network that aggregates nearly 400 sources of data. Sources of data with regards to flow and traffic incidents include: road sensors, traffic cameras, commercial vehicle GPS probes, consumer vehicle GPS probes, cellular network probes, road crashes, and road construction. Once the source-aggregated traffic data is collected, it then gets processed using a proprietary data fusion engine. An overview of the INRIX total fusion engine is presented in Figure 4. INRIX currently covers busy streets, arterials, major freeways, and the entire interstate system. It is combining real-time, historical and predictive traffic data for more than 800,000 miles across the United States (INRIX Inc., 2014).

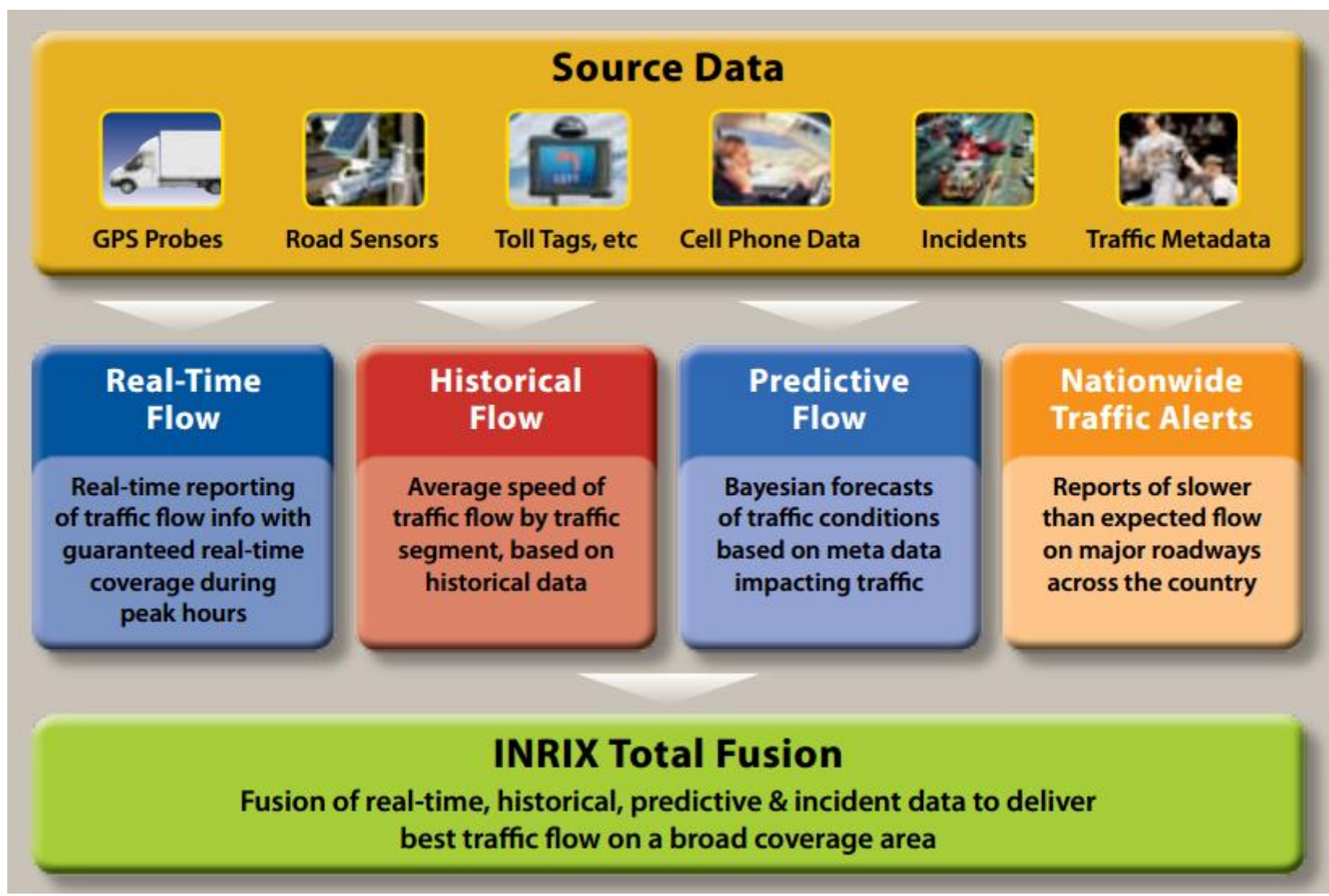

Figure 4: INRIX Fusion Engine

Source: INRIX Inc. 
A corridor in the INRIX data is comprised of multiple segments called Traffic Message Channels (TMCs). Table 4 presents a list of specific TMCs selected for the study area. Performance measures such as real time speed, travel time, and confidence score are recorded for each TMC. The possible confidence score reported for the INRIX readings listed from highest to lowest are 30,20 , and 10 . These three levels are interpreted as following:

- "30" - Completely based on real-time data.

- " $20 "$ - Based on a combination of real-time and historical data.

- "10" - Completely based on historical data.

Table 4: INRIX Traffic Message Channels (TMCs)

\begin{tabular}{|c|c|c|c|c|}
\hline Direction & TMC & Begins & Ends & Length (miles) \\
\hline \multirow{3}{*}{ 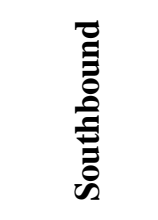 } & $114-07920$ & SW Gaard St & SW Durham & 0.81 \\
\hline & 114-07919 & OR-217 Off-ramp & SW McDonald & 1.68 \\
\hline & $114-07918$ & I-5 Off-ramp & OR-217 Off-ramp & 1.01 \\
\hline \multirow{3}{*}{ 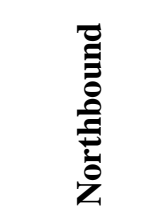 } & $114+07921$ & OR-217 Off-ramp & SW Coronado St & 0.81 \\
\hline & $114+07920$ & SW McDonald St & OR-217 On-Ramp & 1.67 \\
\hline & $114+07919$ & SW Durham St & SW Gaard St & 1.01 \\
\hline
\end{tabular}




\subsection{DATA PROCESSING}

\subsection{Temporal Alignment of Segments}

Since there was a total of 4 Bluetooth segments in each travelling direction and a total of 3 INRIX segments in the study area, The Bluetooth segments had to be reduced to 3 segments. This was accomplished by summing up the travel time of two consecutive Bluetooth segments in each direction to create a new longer segment $r$ by using the following expression:

$$
T T_{r}(t)=\sum_{i=1}^{S} T T_{i}(t) \forall t \in T
$$

Where,

$T T_{i}=$ Average travel time for segment $i$ during time interval $t$

$T T_{r}=$ Average travel time for the new combined segment $r$ during time interval $t$

$S=\{$ Segments to be combined $\}$

\subsection{Spatial Alignment of Segments}

The Bluetooth segments and the INRIX segments were then plotted on a map and it was evident in some areas that the INRIX segment starting and ending points did not fully align with the Bluetooth segment starting and ending points. In order to make a one-to-one comparison, the segments starting and ending points needed to be completely matching. This was resolved by altering the INRIX segments. Figure 5 is a scenario used in explaining the process used to correct for the spatial alignment.

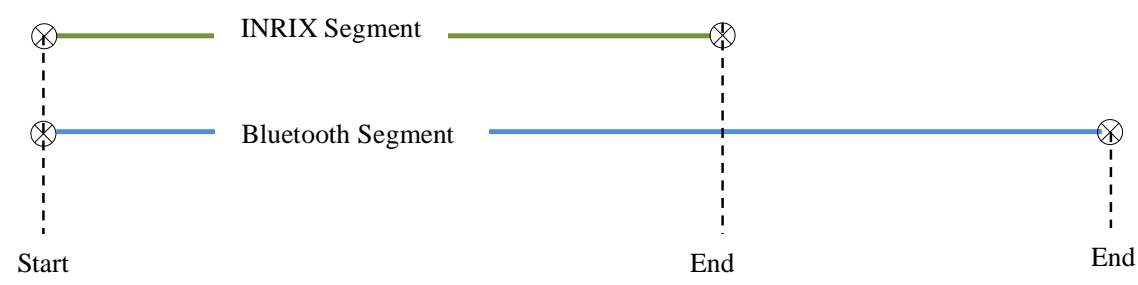

Figure 5: Aligning Start and End Point of Segments 
In the figure a vehicle is expected to take less time to traverse the INRIX segment than the Bluetooth segment thus the travel time data from the INRIX segment cannot be compared to the travel time data from the Bluetooth segment. Given the INRIX average speed $\left(S_{\text {INRIX }}\right)$, the Bluetooth segment length $\left(L_{\text {Bluetooth }}\right)$ and the INRIX segment length $\left(L_{\text {INRIX }}\right)$, the adjusted INRIX travel time (TT Adjusted INRIX) was calculated using the following expression:

$$
\operatorname{TT}_{\text {Adjusted INRIX }}(t)=\operatorname{TT}_{\text {INRIX }}(t)+\frac{L_{\text {Bluetooth }}-L_{\text {INRIX }}}{S_{\text {INRIX }}(t)} \forall t \in T
$$

Where,

$\operatorname{TT}_{\text {INRIX }}(t)=$ Average INRIX travel time before alignment at time interval $t$

The INRIX confidence score described earlier falls into one of three categories (30, 20, and 10) INRIX travel time reported with confidence score of " 20 " and " 10 " were filtered out thus the analysis was evaluated using the real-time INRIX data, which reflects the highest level of confidence. The INRIX data and the Bluetooth data reported travel time information at a one minute interval. In order to directly compare the two data sets with one another, a minute-tominute correspondence was established for all segments in the study area. 


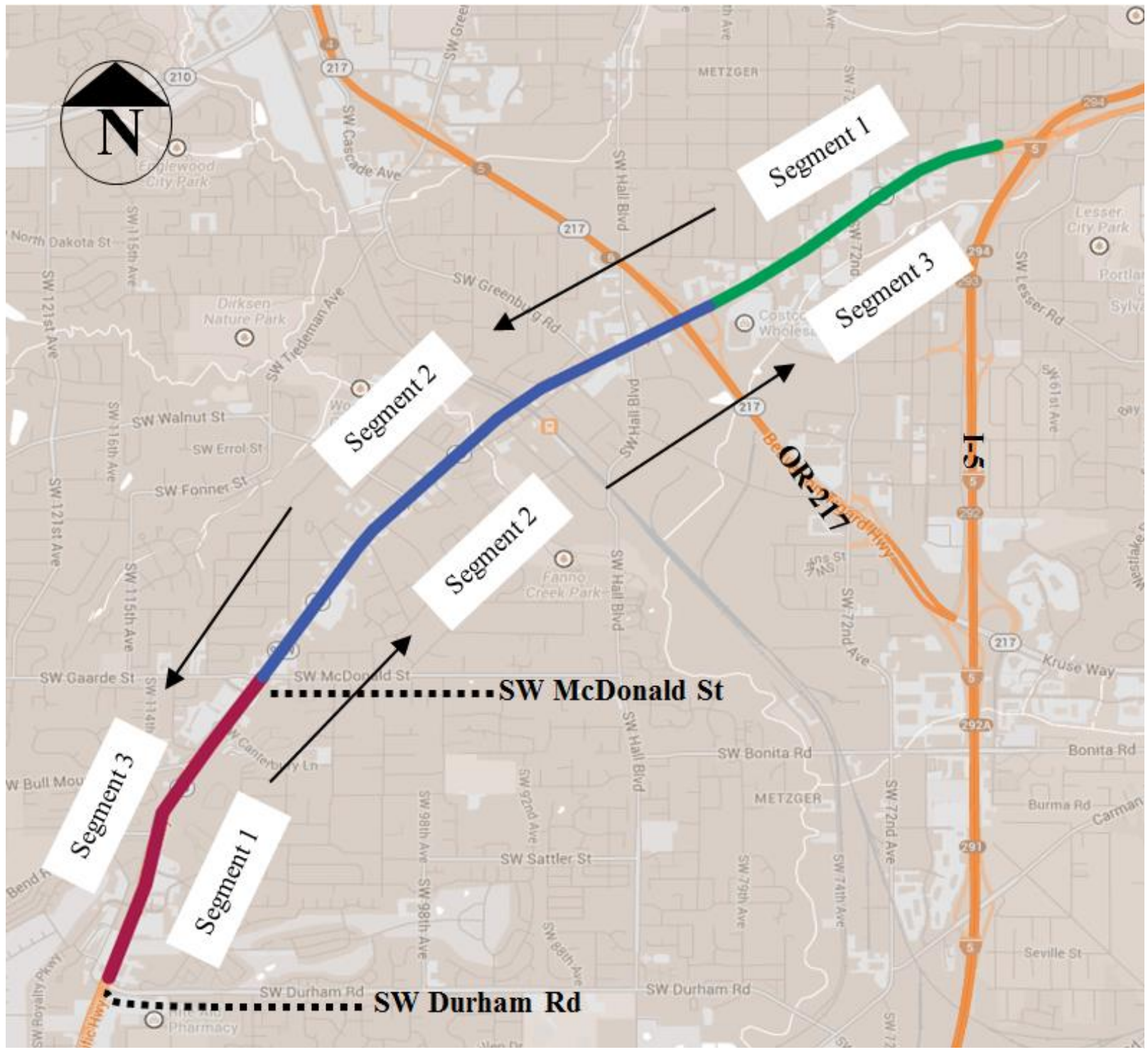

Figure 6: OR 99W Study Segments 


\subsection{RESULTS}

The 4.2-miles of OR 99W corridor was broken down into six segments as shown in Figure 6. For each segment and each time period (Midday, PM Peak, and Weekend), the Bluetooth data as well as the INRIX data were compared to the benchmark. An example of the post-processed data is shown in Figure 7. The figure shows the travel time estimates of the Bluetooth system plotted in blue, the INRIX system plotted in green, and the probe travel times for the same traversals as black squares for one day, April 1, 2014. Similar data were used to compare each probe run was paired with its equivalent Bluetooth and INRIX reading. The basis of the comparison for the Bluetooth-to-probe data and INRIX-to-probe data were quantitative (statistical) and qualitative (graphical). For the quantitative analysis, the mean absolute error (MAE) in minutes and the mean absolute percent error (MAPE) values were calculated for each segment and time period. The MAPE values were produced using the following procedure:

1. Each probe run is paired with its equivalent estimated travel time (Bluetooth and INRIX).

2. The difference between the probe travel time and the estimated travel time is then divided by the probe travel time to calculate the percent error.

3. The absolute value of the percent error is then average over each time period (midday, pm peak, and weekend) to create a single MAPE value for that time period.

Since the MAPE value shows the magnitude of the error but fails to show the direction of the error, the average error in minutes was calculated for each time period of each segment. Based on the direction of the error (positive or negative), the following categories were created:

a) Overestimated

- Bluetooth travel time $>$ probe travel time.

- INRIX travel time $>$ probe travel time.

b) Underestimated

- Bluetooth travel time $<$ probe travel time.

- INRIX travel time $\quad<$ probe travel time 

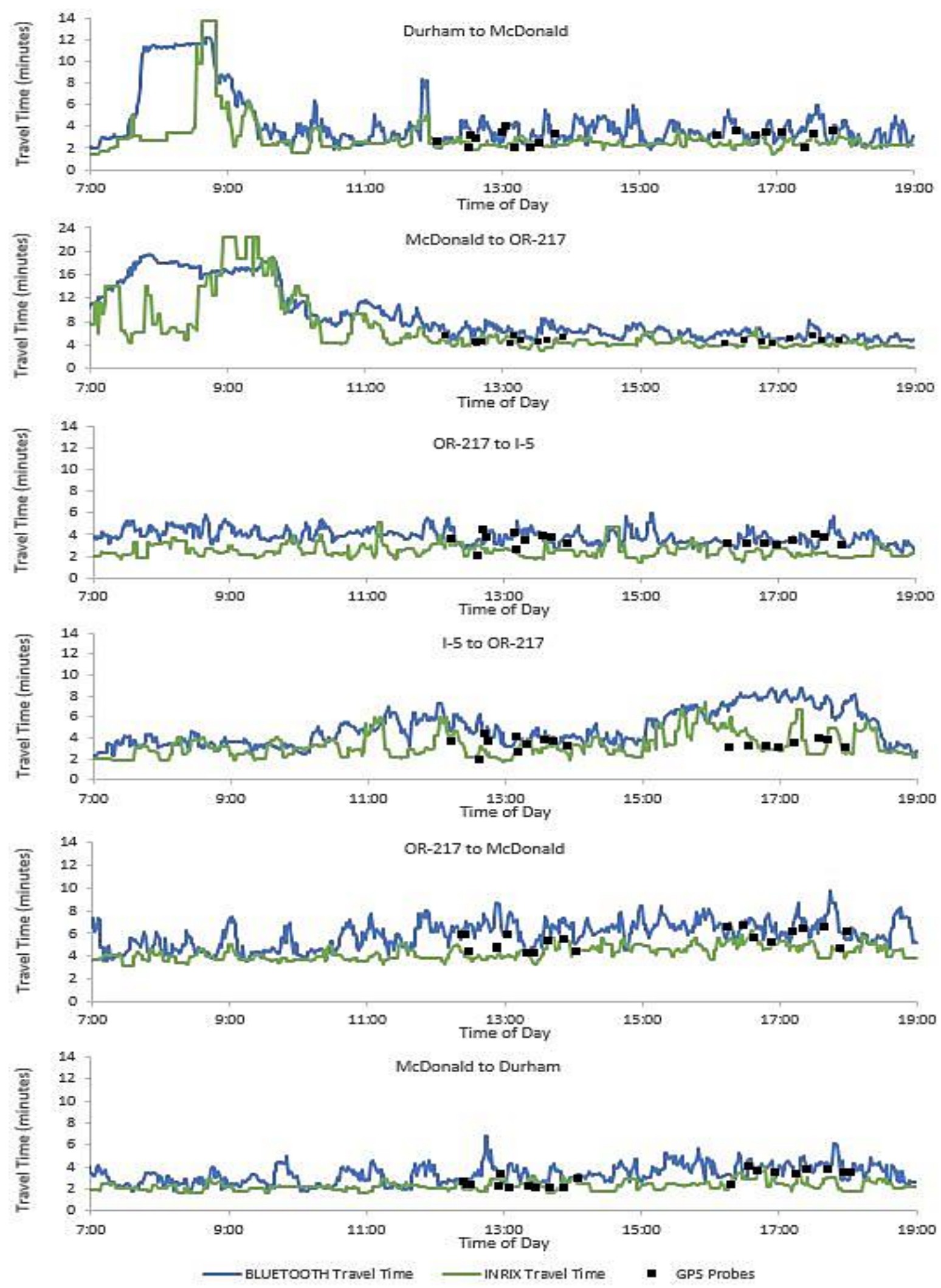

Figure 7: Sample Time Series Plots (4/1/2014) 
Furthermore, statistical hypothesis tests (Equations 3 and 4) for all segments and time periods were conducted to evaluate the accuracy of travel time estimates. Unlike the recorded travel time of the probe vehicle that was based on a single vehicle, the Bluetooth and the INRIX travel time are averages of multiple vehicles for a single time interval. Therefore, the results obtained from the hypothesis test were considered of a high bar. The null hypothesis in these statistical tests state that there is no difference between the mean travel time estimates ( $\mu_{\text {Bluetooth }}$ and $\mu_{\text {INRIX }}$ ) and the mean probe travel time $\left(\mu_{\text {Probe }}\right)$.
(Null Hypothesis)
$H_{0}: \mu_{\text {Bluetooth }}-\mu_{\text {Probe }}=0$
(Alternative Hypothesis)
$H_{1}: \mu_{\text {Bluetooth }}-\mu_{\text {Probe }} \neq 0$
(Null Hypothesis)

$$
H_{0}: \mu_{I N R I X}-\mu_{\text {Probe }}=0
$$$$
\text { (Alternative Hypothesis) } \quad H_{1}: \mu_{I N R I X}-\mu_{\text {Probe }} \neq 0
$$

The statistical hypothesis testing was performed at a level of confidence ( $\alpha$ ) equal to 0.05 .

\subsection{Bluetooth - Probe Comparison}

The results of the Bluetooth comparison to the probe data is shown in Table 5. The mean absolute error results show that the Bluetooth travel time is most accurate during the pm peak period and least accurate during the midday period. The average error ranges from a low of 0.61 minutes for the Durham to McDonald section on the weekend to a high of 1.94 minutes for the McDonald to OR-217 section on the weekend.

It is important to note that the segments are relatively short (approximately 1 mile), thus the percent error can be high. Based on the MAPE values, the travel time estimates during the pm peak period is most accurate and the weekend period is least accurate. The MAPE value ranges from a low of $11.86 \%$ for OR-217 to McDonald segment on the weekend to a high of $57.26 \%$ for I-5 to OR-217 section on weekend.

To find the direction of the error (overestimated or underestimated), the average error values were calculated and the overestimated runs were separated from the underestimated runs. The percent of overestimated runs were calculated by dividing the number of overestimate runs by 
the total number of runs. The results show that the majority of the Bluetooth estimates were overestimated.

At a $95^{\text {th }}$ confidence level, a p-value less than 0.05 indicates that there was a statistical significance difference in travel time between the Bluetooth data and the ground truth data. Likewise, p-values larger than 0.05 indicate insufficient evidence to conclude a difference. The pm peak travel times are most accurate and the midday travel times are least accurate. During the pm peak period only 2 out 6 segments witnessed a significant difference.

\subsection{INRIX - Probe Comparison}

The results of the INRIX comparison to the probe data is shown in Table 5. The mean absolute error results show that the INRIX travel time was most accurate during the Midday with all segments having mean absolute error of less than a minute. The average error ranges from a low of 0.15 minutes for the McDonald to OR-217 section on the Midday to a high of 7.6 minutes for the McDonald to OR-217 section on the weekend. Moreover, the Durham to McDonald segment experienced accurate travel time for all time periods.

Based on the MAPE values, the midday period is most accurate having 5 out of 6 segments with MAPE value less than 25\%. The MAPE value ranges from a low of $11.86 \%$ for OR-217 to McDonald segment on the weekend to a high of $57.26 \%$ for I-5 to OR-217 section on weekend. In addition, the OR-217 to McDonald segment experiences an MAPE value less of less than 25\% across all time periods.

The overestimation percentage results indicate that the INRIX estimates were all underestimated. Moreover, results of the p-value from the matched pairs t-test show the midday estimates to be most accurate, while the weekend estimates to be least accurate. Table 5 represents a summary of the statistical measures discussed for both the Bluetooth and the INRIX travel time estimates. 
Table 5: Summary of Statistical Measures for (Bluetooth \& INRIX) to Probe

\begin{tabular}{|c|c|c|c|c|c|c|c|c|}
\hline \multicolumn{9}{|c|}{ Mean Absolute Error in Minutes $(M A E<1.00$ shaded $)$} \\
\hline \multirow{2}{*}{ Direction } & \multirow{2}{*}{ Segment } & \multirow{2}{*}{ Segment Name } & \multicolumn{3}{|c|}{ Bluetooth } & \multicolumn{3}{|c|}{ INRIX } \\
\hline & & & Midday & PM Peak & Weekend & Midday & PM Peak & Weekend \\
\hline \multirow{3}{*}{ Southbound } & 1 & I-5 to OR-217 & 1.18 & 1.18 & 1.36 & 0.21 & 2.83 & 0.76 \\
\hline & 2 & OR-217 to McDonald & 1.54 & 1.16 & 0.68 & 0.16 & 1.35 & 1.42 \\
\hline & 3 & McDonald to Durham & 1.08 & 0.74 & 1.03 & 0.21 & 1.34 & 0.63 \\
\hline \multirow{3}{*}{ Northbound } & 1 & Durham to McDonald & 1.05 & 0.92 & 0.61 & 0.69 & 0.87 & 0.92 \\
\hline & 2 & McDonald to OR-217 & 1.32 & 0.98 & 1.94 & 0.15 & 0.91 & 7.60 \\
\hline & 3 & OR-217 to I-5 & 0.85 & 0.62 & 0.92 & 0.36 & 0.87 & 1.10 \\
\hline \multicolumn{9}{|c|}{ Mean Absolute Percent Error (MAPE <25\% shaded) } \\
\hline \multirow{2}{*}{ Direction } & \multirow{2}{*}{ Segment } & \multirow{2}{*}{ Segment Name } & \multicolumn{3}{|c|}{ Bluetooth } & \multicolumn{3}{|c|}{ INRIX } \\
\hline & & & Midday & PM Peak & Weekend & Midday & PM Peak & Weekend \\
\hline \multirow{3}{*}{ Southbound } & 1 & I-5 to OR-217 & $44.4 \%$ & $25.2 \%$ & $57.6 \%$ & $21.5 \%$ & $39.9 \%$ & $27.3 \%$ \\
\hline & 2 & OR-217 to McDonald & $31.9 \%$ & $22.4 \%$ & $11.9 \%$ & $15.9 \%$ & $20.6 \%$ & $24.5 \%$ \\
\hline & 3 & McDonald to Durham & $47.7 \%$ & $16.1 \%$ & $46.4 \%$ & $21.1 \%$ & $33.1 \%$ & $21.9 \%$ \\
\hline \multirow{3}{*}{ Northbound } & 1 & Durham to McDonald & $45.7 \%$ & $41.7 \%$ & $19.5 \%$ & $24.3 \%$ & $34.3 \%$ & $26.5 \%$ \\
\hline & 2 & McDonald to OR-217 & $27.2 \%$ & $20.9 \%$ & $13.2 \%$ & $15.0 \%$ & $17.6 \%$ & $54.1 \%$ \\
\hline & 3 & OR-217 to I-5 & $30.1 \%$ & $20.3 \%$ & $31.7 \%$ & $36.3 \%$ & $26.7 \%$ & $30.7 \%$ \\
\hline \multicolumn{9}{|c|}{ Percent of Overestimated Travel Time (Overestimate > 50\% shaded) } \\
\hline \multirow{2}{*}{ Direction } & \multirow{2}{*}{ Segment } & \multirow{2}{*}{ Segment Name } & \multicolumn{3}{|c|}{ Bluetooth } & \multicolumn{3}{|c|}{ INRIX } \\
\hline & & & Midday & PM Peak & Weekend & Midday & PM Peak & Weekend \\
\hline \multirow{3}{*}{ Southbound } & 1 & I-5 to OR-217 & $100 \%$ & $59 \%$ & $90 \%$ & $13 \%$ & $0 \%$ & $30 \%$ \\
\hline & 2 & OR-217 to McDonald & $75 \%$ & $71 \%$ & $75 \%$ & $0 \%$ & $0 \%$ & $13 \%$ \\
\hline & 3 & McDonald to Durham & $87 \%$ & $41 \%$ & $89 \%$ & $33 \%$ & $0 \%$ & $22 \%$ \\
\hline \multirow{3}{*}{ Northbound } & 1 & Durham to McDonald & $71 \%$ & $50 \%$ & $89 \%$ & $36 \%$ & $44 \%$ & $11 \%$ \\
\hline & 2 & McDonald to OR-217 & $100 \%$ & $75 \%$ & $33 \%$ & $29 \%$ & $6 \%$ & $0 \%$ \\
\hline & 3 & OR-217 to I-5 & $79 \%$ & $63 \%$ & $78 \%$ & $14 \%$ & $6 \%$ & $0 \%$ \\
\hline \multicolumn{9}{|c|}{ P-Values for Matched Pairs T-Test $(P$-Value $>0.05$ shaded $)$} \\
\hline \multirow{2}{*}{ Direction } & \multirow{2}{*}{ Segment } & Coratom & & Bluetooth & & & INRIX & \\
\hline & & segment Name & Midday & PM Peak & Weekend & Midday & PM Peak & Weekend \\
\hline & 1 & I-5 to OR-217 & 0.00 & 0.47 & 0.01 & 0.45 & 0.00 & 0.79 \\
\hline Southbound & 2 & OR-217 to McDonald & 0.01 & 0.01 & 0.08 & 0.00 & 0.00 & 0.12 \\
\hline & 3 & McDonald to Durham & 0.01 & 0.43 & 0.03 & 0.48 & 0.00 & 0.35 \\
\hline & 1 & Durham to McDonald & 0.02 & 0.06 & 0.06 & 0.07 & 0.59 & 0.07 \\
\hline Northbound & 2 & McDonald to OR-217 & 0.00 & 0.01 & 0.09 & 0.22 & 0.00 & 0.00 \\
\hline & 3 & OR-217 to I-5 & 0.04 & 0.08 & 0.03 & 0.01 & 0.00 & 0.00 \\
\hline
\end{tabular}




\subsection{Bluetooth - INRIX Comparison}

In Figure 8, the matched pairs of the travel time runs for all of the segments and time periods evaluated are shown. In the Figure, the y-axis represents the probe travel time and the $\mathrm{x}$-axis represents the crowd sourced travel time. The Bluetooth estimates are shown in blue; the INRIX estimates in green. If all estimates were equal, they would fall on the dashed line in the figure. The plots reinforce the analysis in Table 5, that the INRIX data that tends to underestimate travel times and the Bluetooth data tends to overestimate travel times.

\section{Difference in Travel Time Means}

To determine whether the travel time obtained from the Bluetooth data was similar or different from the travel time obtained from the INRIX data, a matched-pairs t-test was conducted. A total of 13,541 observation from three days (4/1/2014, 4/3/2014, and 4/5/2014) were used as an input for the matched-pairs t-test. The null hypothesis in the statistical test (Equation 5) was that there was no difference between the Bluetooth mean travel time $\left(\mu_{\text {Bluetooth }}\right)$ and the INRIX mean travel time $\left(\mu_{I N R I X}\right)$ in each time interval.

(Null Hypothesis)

(Alternative Hypothesis)

$$
\begin{aligned}
& H_{0}: \mu_{\text {Bluetooth }}-\mu_{\text {INRIX }}=0 \\
& H_{1}: \mu_{\text {Bluetooth }}-\mu_{\text {INRIX }} \neq 0
\end{aligned}
$$

The statistical hypothesis testing was performed at a level of confidence ( $\alpha$ ) equal to 0.05 . The matched pairs t-test for the entire dataset (13,541 observations) showed sufficient evidence to conclude that the difference between the Bluetooth mean travel time and the INRIX mean travel time was significant. The mean of the differences was found to be 1.87 minutes, thus suggesting the Bluetooth mean travel time was significantly higher than the INRIX mean travel time. The results of the hypothesis test is presented in Table 6

\begin{tabular}{|c|c|c|c|c|c|c|c|}
\hline \multirow{3}{*}{ Pair } & \multicolumn{4}{|c|}{ Paired Differences } & \multirow{3}{*}{ T stat } & \multirow{3}{*}{ df } & \multirow{3}{*}{$\begin{array}{l}\text { P-value } \\
\text { (2-tailed) }\end{array}$} \\
\hline & \multirow{2}{*}{ Mean } & \multirow{2}{*}{$\begin{array}{l}\text { Standard } \\
\text { Deviation }\end{array}$} & \multicolumn{2}{|c|}{ 95\% Confidence Interval } & & & \\
\hline & & & Lower & Upper & & & \\
\hline$\mu_{\text {Bluetooth }}-\mu_{\text {INRIX }}$ & 1.867 & 1.589 & 1.840 & 1.894 & 136.67 & 13540 & 0.00 \\
\hline
\end{tabular}

Table 6: Matched Pairs T-Test for the Difference in Means 
Durham to McDonald
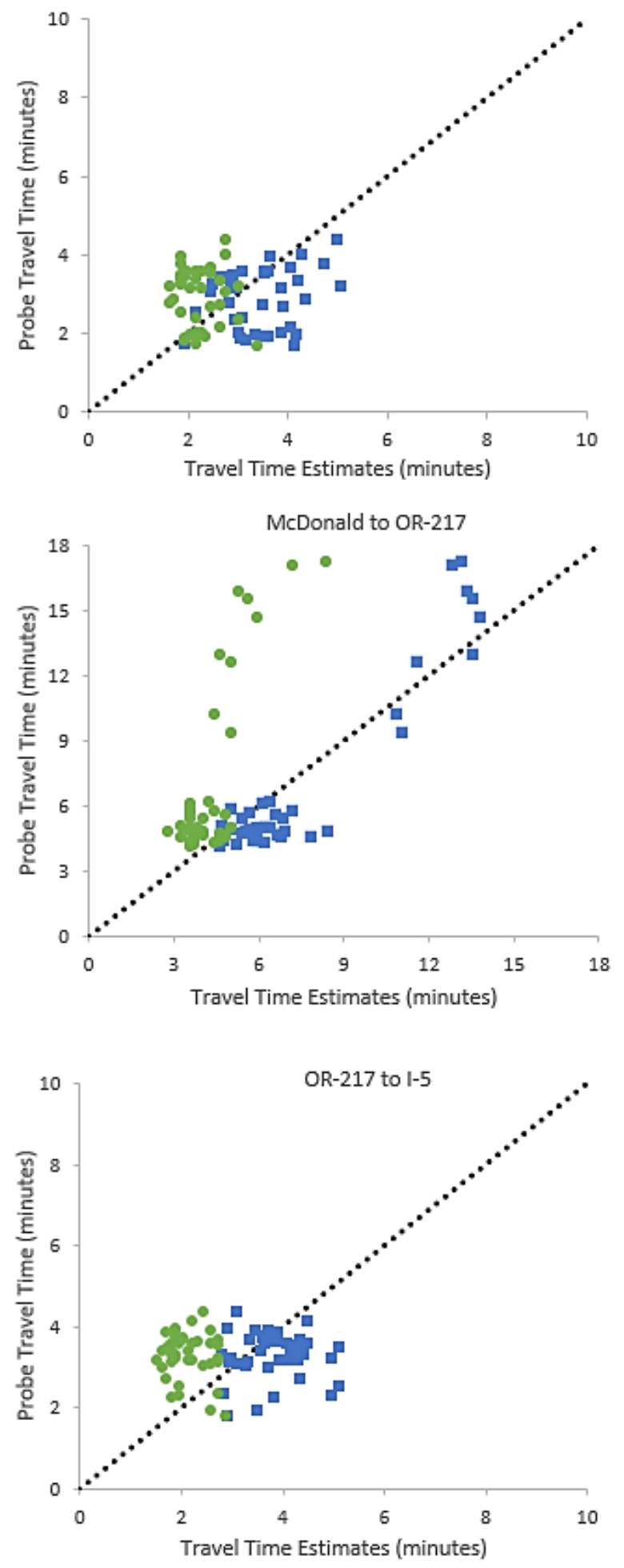

I-5 to OR-217
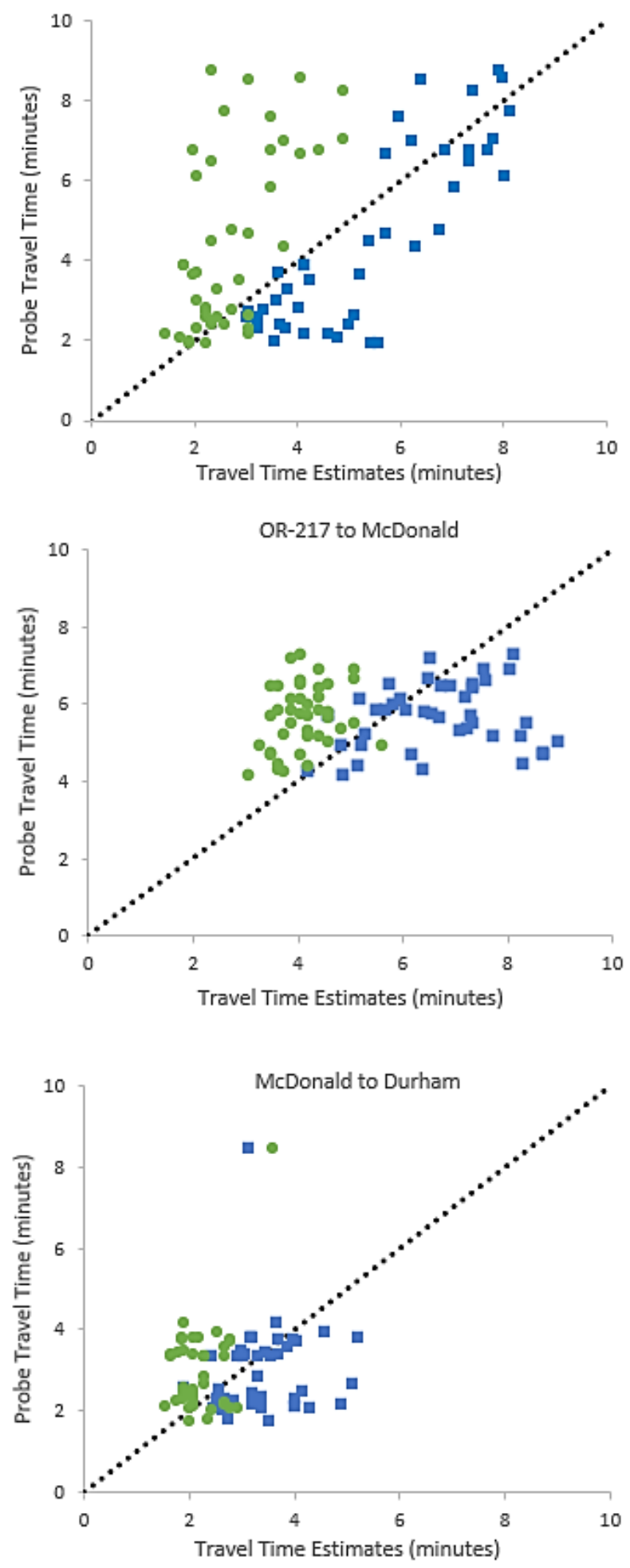

口 Bluetooth Travel Time

- INRIX Travel Time

...... Ideal Travel Time

Figure 8: Probe Travel Time vs Estimates Travel Time 


\section{Correlations}

In Figure 9, a sample of time series plot is shown for the INRIX and the Bluetooth data. In the figure the $y$-axis represents the travel time estimate and the $\mathrm{x}$-axis represents the time of day. The bluetooth estimates are shown in blue, and the INRIX estimates are shown in green. It can be noticed from the figure that the both data sets have a relatively matching increasing and decreasing trends.

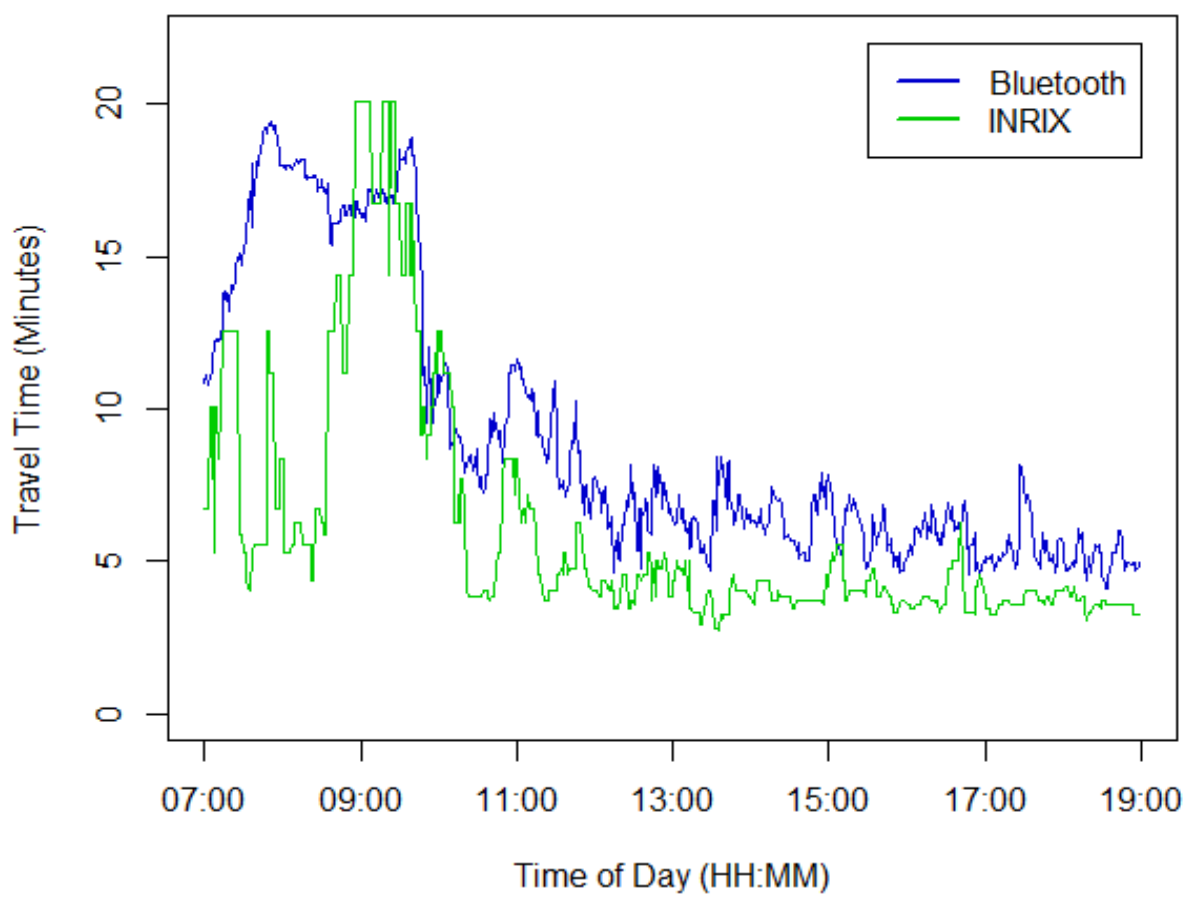

Figure 9: Time Series Profile of McDonald to Durham (4/1/2014)

A correlation is a dimensionless statistical measure of linear association between a pair of variables. The correlation takes on a value between -1 and +1 . A value of 0 indicates no linear association, while a -1 and +1 indicate a perfect linear association. A positive value indicates a positive linear association, likewise a negative value indicates a negative linear association. The correlation of a population $(\rho)$ is expressed by:

$$
\rho(x, y)=\frac{\operatorname{Cov}(x, y)}{\sigma_{x} \sigma_{y}}
$$


Where,

Cov $=$ Covariance of the pair $(x, y)$

$\sigma_{\mathrm{x}}=$ Standard deviation of $x$

$\sigma_{\mathrm{y}} \quad=$ Standard deviation of $y$

To better understand the magnitude of the similarity in trends, a hypothesis test for the population correlation $(\rho)$ was conducted. The null hypothesis in the statistical test (Equation 7) was that correlation between the Bluetooth and INRIX pairs is equal to zero.

(Null Hypothesis)

$H_{0}: \rho=0$

(Alternative Hypothesis)

$$
H_{1}: \rho \neq 0
$$

At a $95^{\text {th }}$ confidence level, a p-value less than 0.05 indicates that that the Bluetooth time series is correlation to the INRIX time series. Likewise, p-values larger than 0.05 indicate insufficient evidence to conclude an existence of a correlation. The results of the hypothesis test showed sufficient evidence to conclude that the Bluetooth and INRIX time series are correlated for all segments and days with the exception of McDonald to Durham section on Saturday, and OR-217 to I-5 section on Tuesday and Saturday. The p-values for all segments by day are summarized in Table 7.

Table 7: P-Values for the Correlation Hypothesis testing $(P$-Value $>0.05$ shaded $)$

\begin{tabular}{|c|c|c|c|c|c|}
\hline \multirow{2}{*}{ Direction } & \multirow{2}{*}{ Segment } & \multirow{2}{*}{ Segment Name } & \multicolumn{3}{|c|}{ Time Period $(7: 00-19: 00)$} \\
\hline & & & Tuesday & Thursday & Saturday \\
\hline \multirow{3}{*}{ Southbound } & 1 & I-5 to OR-217 & 0.00 & 0.00 & 0.00 \\
\hline & 2 & OR-217 to McDonald & 0.00 & 0.00 & 0.00 \\
\hline & 3 & McDonald to Durham & 0.00 & 0.00 & 0.05 \\
\hline \multirow{3}{*}{ Northbound } & 1 & Durham to McDonald & 0.00 & 0.00 & 0.00 \\
\hline & 2 & McDonald to OR-217 & 0.00 & 0.00 & 0.00 \\
\hline & 3 & OR-217 to I-5 & 0.11 & 0.00 & 0.51 \\
\hline
\end{tabular}


The cross correlation function $(\mathrm{CCF})$ is another approach used to determine the correlation between two time series. The CCF produces a plot to check for lagged correlation between the Bluetooth and the INRIX time series. Figure 10 is an example of a CCF correlogram for the Bluetooth and the INRIX time series. In the figure, the INRIX is shown to be lagging the Bluetooth estimates and the strongest association takes place at lag -2 with a correlation of 0.78 .

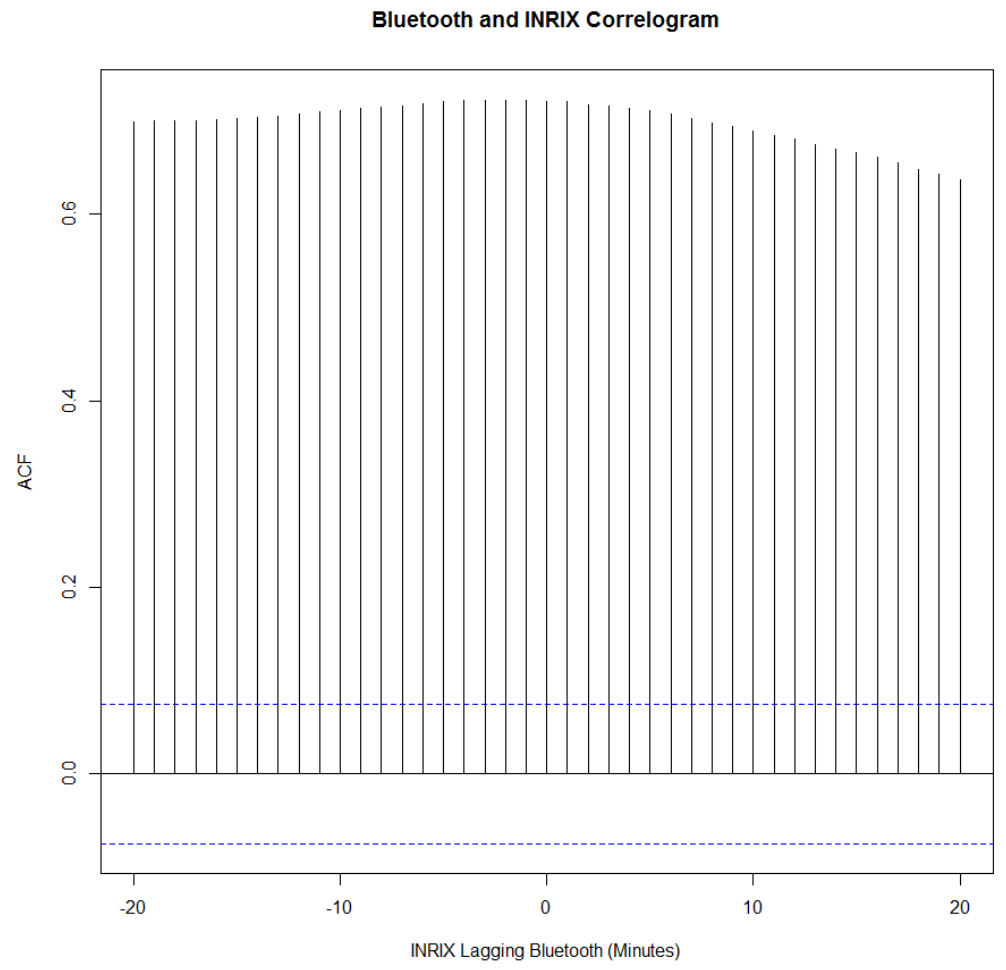

Figure 10: CCF Correlogram for McDonald to OR-217 (4/1/2014)

A summary of the correlations for all segments and all days is presented in Table 8. In the table strong correlations $(\rho \geq 0.40)$ are shaded in green, moderate correlations $(0.19<\rho<0.49)$ are shaded in yellow and negligible correlations $(\rho \leq 0.19)$ are shaded in red.

Table 8: Cross Correlation for all Segments and Days

\begin{tabular}{c|c|l|c|c|c}
\hline \multirow{2}{*}{ Direction } & \multirow{2}{*}{ Segment } & \multirow{2}{*}{ Segment Name } & \multicolumn{3}{|c}{ Time Period (7:00 - 19:00) } \\
\cline { 4 - 6 } & & & Tuesday & Thursday & Saturday \\
\hline \multirow{3}{*}{ Southbound } & 1 & I-5 to OR-217 & 0.49 & 0.59 & 0.47 \\
\cline { 2 - 6 } & 2 & OR-217 to McDonald & 0.33 & 0.51 & 0.60 \\
\cline { 2 - 6 } & 3 & McDonald to Durham & 0.23 & 0.20 & 0.08 \\
\hline \multirow{3}{*}{ Northbound } & 1 & Durham to McDonald & 0.58 & 0.39 & 0.28 \\
\cline { 2 - 6 } & 2 & McDonald to OR-217 & 0.72 & 0.33 & 0.57 \\
\cline { 2 - 6 } & 3 & OR-217 to I-5 & 0.06 & 0.24 & 0.02 \\
\hline
\end{tabular}




\subsection{CONCLUSIONS}

In this research the INRIX travel time data was compared to the traditional Bluetooth travel time estimates. The INRIX data was found to be most accurate during the midday period, while the Bluetooth data was found most accurate during the pm peak period. The INRIX estimates during the midday were either within 0.36 minutes or $22 \%$ of the ground truth probe runs. The Bluetooth estimates during the pm peak were either within 1 minute or $24 \%$ of the ground truth probe runs. Unlike the INRIX data that tends to underestimate travel times, the Bluetooth data tends to overestimate travel times.

The matched pairs t-test for 13,541 observations showed the Bluetooth estimates to be significantly different from the INRIX estimates. The hypothesis test for the population correlation $(\rho)$ showed sufficient evidence to conclude that the Bluetooth and INRIX time series are correlated for almost all segments and days. The CCF correlograms validated the existence of a moderate to strong correlation when the INRIX was lagging the Bluetooth estimates. The result of this study demonstrated that satisfying accurate travel time estimates could be obtained from both the Bluetooth and the INRIX datasets.

From this study, it is suggested that future research need to be conducted on other corridor with different characteristics. This study was limited by its focus on three days' worth of data, which could be better improved in terms of confidence by expanding on the size and number of days for the collected data. The merging of the INRIX and the Bluetooth dataset is a promising futuristic step towards improving the accuracy and reliability of travel time estimation. 


\subsection{REFERENCES}

Araghi, Bahar N., Kristian S. Pedersen, Lars T. Christensen, Rajesh Krishnan, and Harry Lahrmann. (2012) Accuracy of Travel Time Estimation Using Bluetooth Technology: A Case Study Limfjord Tunnel Aalborg. 19th ITS World Congress.

Athey Creek Consultant. (2012). Evaluation of Arterial Real-Traveler Information Commercial Probe Data Project. Prepared for MnDOT.

Federal Highway Administration (FHWA). (1998). Travel Time Data Collection Handbook. FHWA-PL98-035.

Haghani A., Hamedi M., and Sadabadi K. (2009) I-95 Corridor Coalition Vehicle Probe Project: Validation of INRIX Data July-September 2008. University of Maryland. College Park, MD.

Haghani, A., Hamedi, M., Sadabadi, K.F., Young, S.E., and Tarnoff, P.J. (2010) Freeway Travel Time Ground Truth Data Collection Using Bluetooth Sensors. In Proceedings 89th Annual Meeting Transportation Research Board, Washington.

INRIX Incorporated. INRIX Total Fusion. http://www.inrix.com/pdf/INRIX\%20Total\%20Fusion.pdf. Accessed July 20, 2014.

Izadpanah, P., and Hellinga, B. (2007). Wide-Area Wireless Traffic Condition Monitoring: Reality or Wishful Thinking?, the 2007 CITE Annual Conference in Toronto, Ontario.

Izadpanah, P., (2010). Freeway Travel Time Prediction Using Data from Mobile Probes. Ph.D. Thesis, Department of Civil and Environmental Engineering, University of Waterloo,Ontario.

Mainovskiy, Y., Wu, Y.J., Wang, Y., Lee, U.K. (2009). Field Experiments on Bluetooth-Based travel time data collection. In Preceedings $89^{\text {th }}$ Annual Meeting Transportation Research Board, Washington.

Oregon Department of Transportation (ODOT) Transportation Data Unit. (2013). 2012 Transportation Volume Tables.

Porter, J. D., Kim, D. S., and Magana, M. E. (2011) Wireless Data Collection System for RealTime Arterial Travel Time Estimates (No. OR-RD-11-10).

Quayle, S., Koonce P., J.V., Bullock, D.M., and DePencier, D. (2010). Arterial Performance Measures Using MAC Readers: Pilot Study in Portland Oregon. In Proceedings 89th Annual Meeting Transportation Research Board, Washington.

Tantiyanugulchai, S. (2004). Arterial Performance Measurement Using Transit Buses as Probe Vehicles. M.S. Thesis, Department of Civil and Environmental Engineering, Portland State University, OR. 
Toppen A and Wunderlich K. (2003). Travel time data collection for measurement of advanced Traveler information systems accuracy. Federal Highway Administration, Project No. 0900610-D1. 20 p.

Washington Department of Transportation (WSDOT) (2014). Error Assessment for Emerging Traffic Data Collection Devices (WA-RD 810.1).

Wasson, J., Sturdevant, J., and Bullock, D.M. (2008). Real-Time Travel Time estimates using Media Access Control Address Matching, ITE Journal, vol. 78, pp.20-23. 
APPENDICES 
APPENDIX A

Travel Time Profiles 

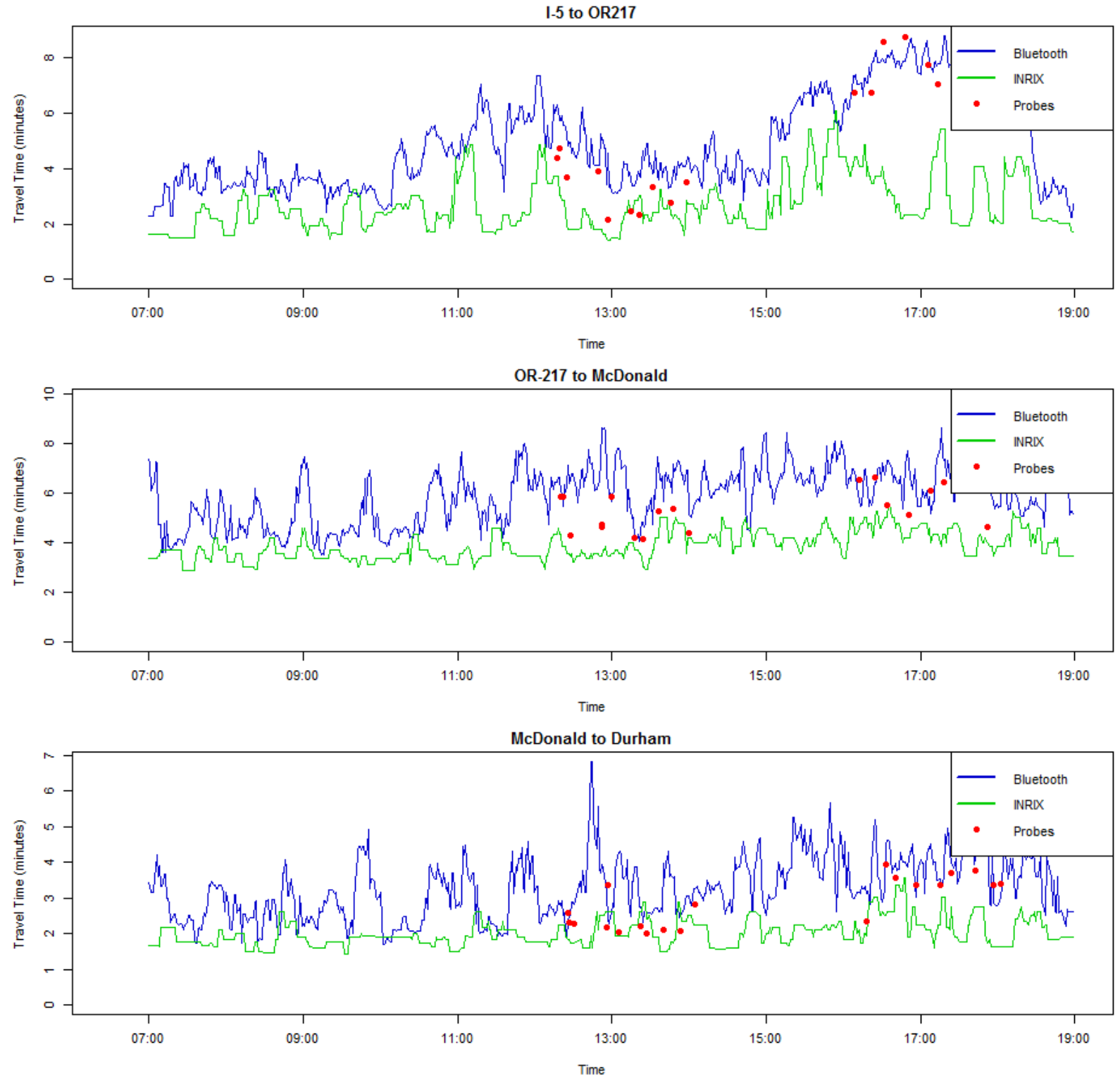

Figure 11: Travel Time Profiles for Southbound Segments (4/1/2014) 

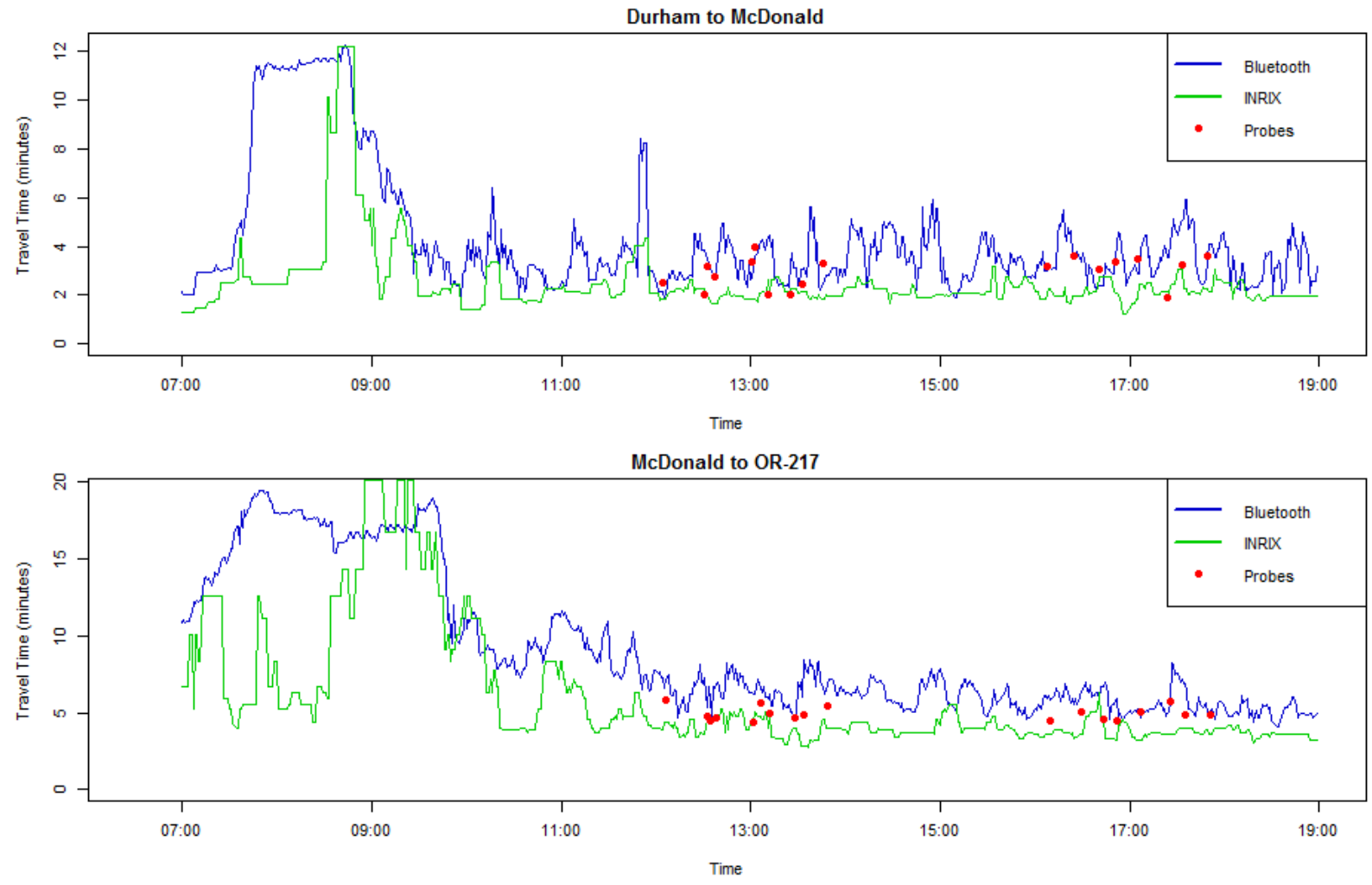

OR217 to I-5

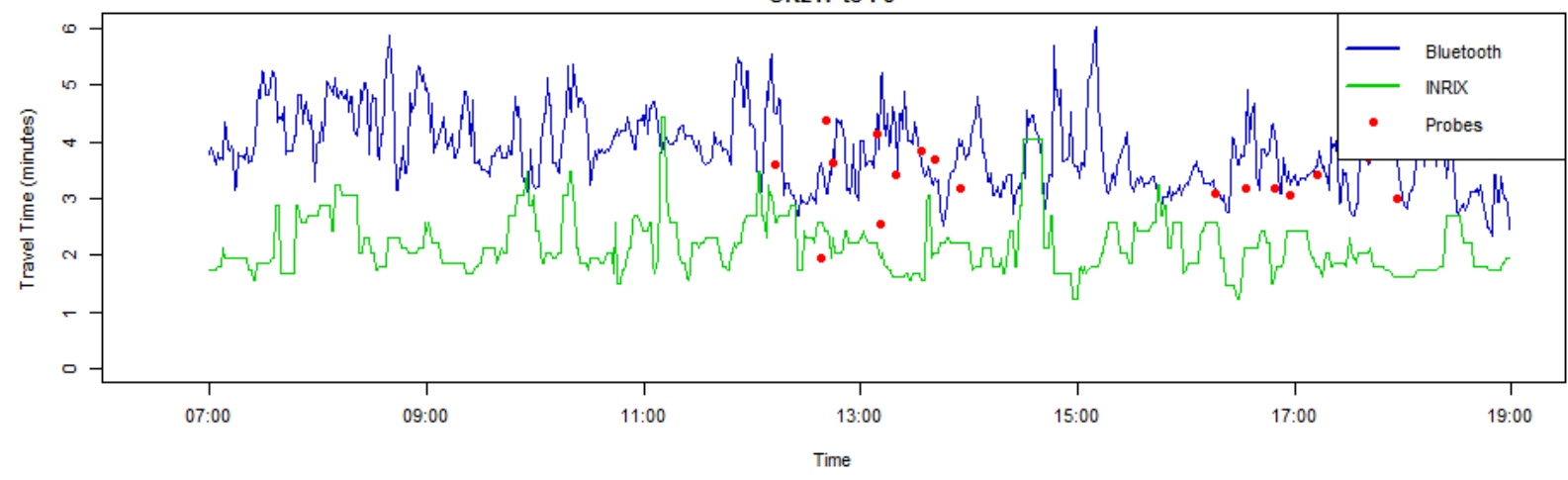

Figure 12: Travel Time Profiles for Northbound Segments (4/1/2014) 

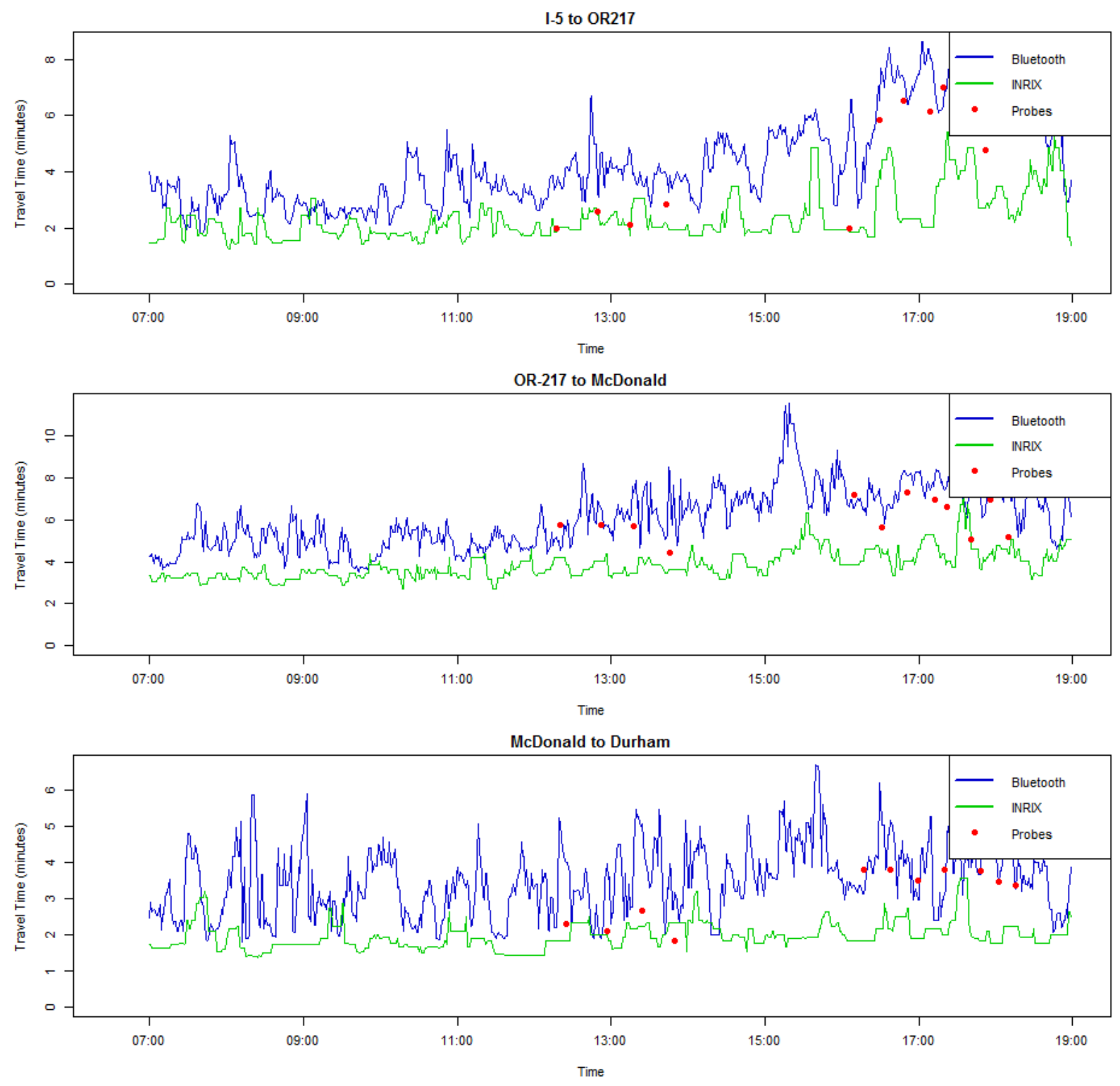

Figure 13: Travel Time Profiles for Southbound Segments (4/3/2014) 

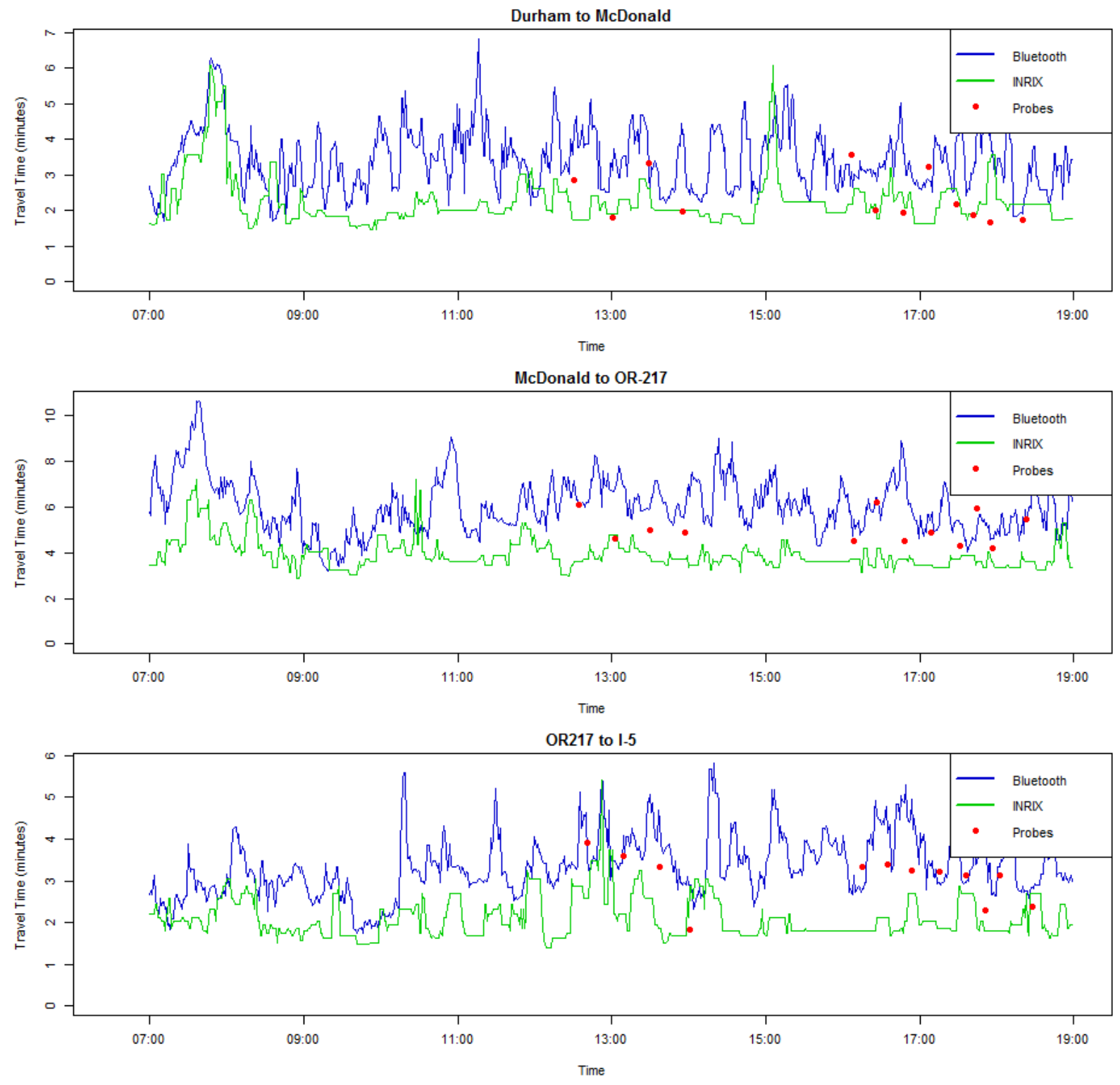

Figure 14: Travel Time Profiles for Northbound Segments (4/3/2014) 

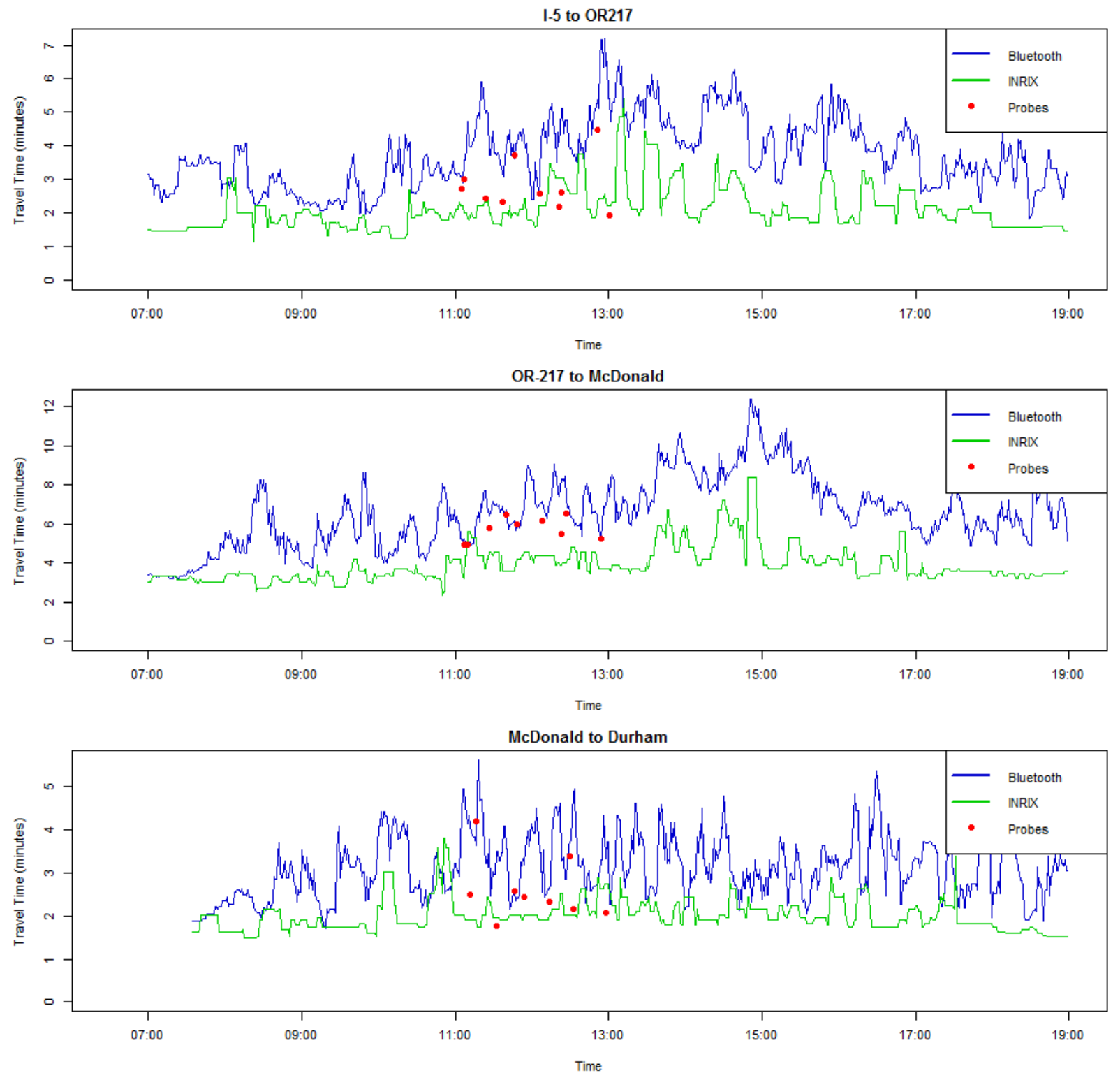

Figure 15: Travel Time Profiles for Southbound Segments (4/5/2014) 

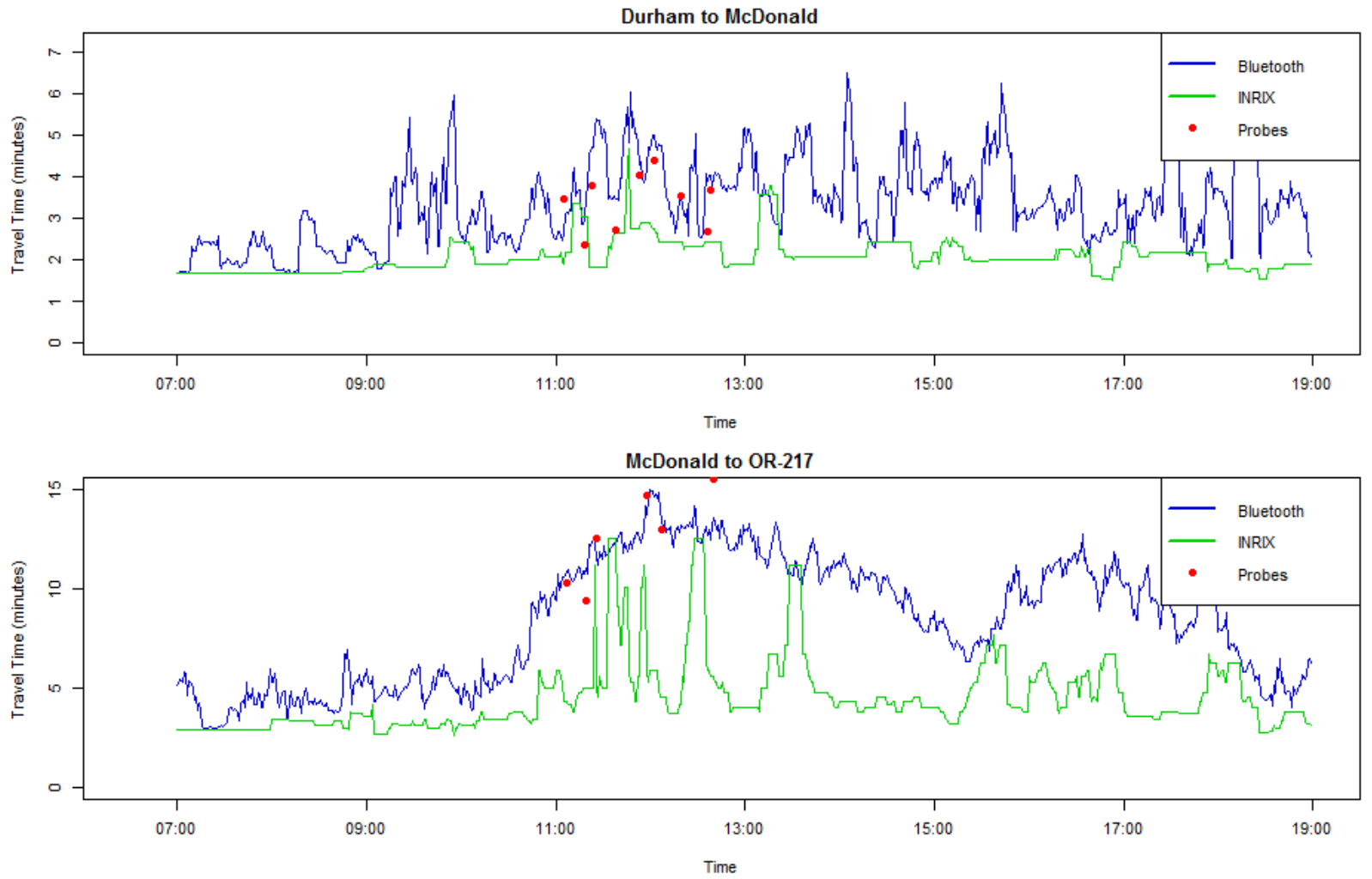

OR217 to I-5

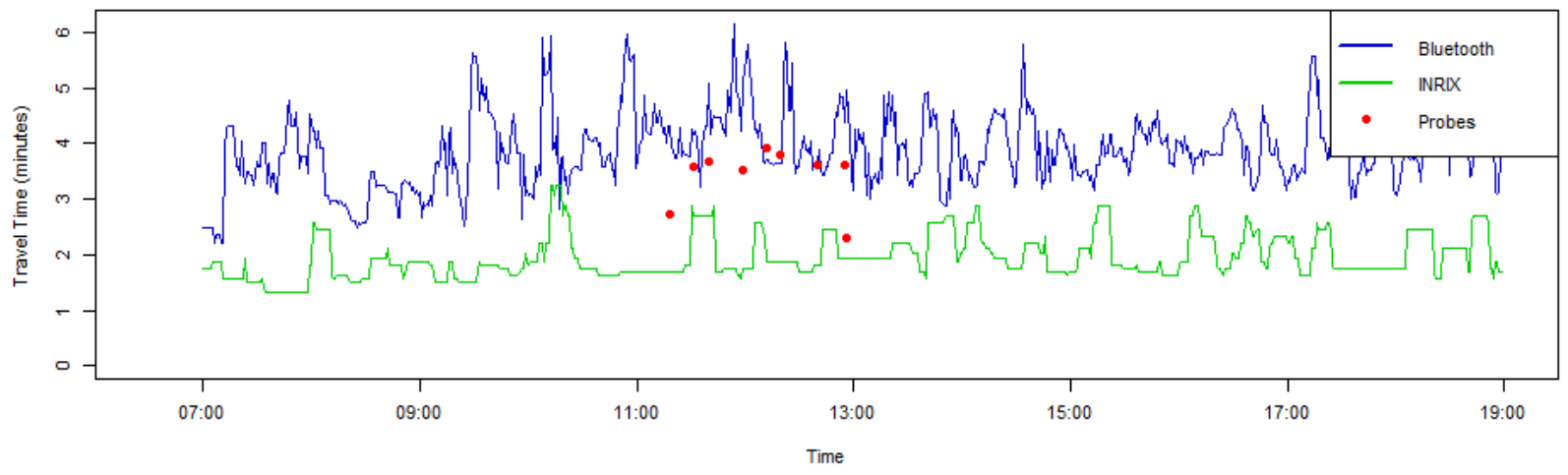

Figure 16: Travel Time Profiles for Northbound Segments (4/5/2014) 
APPENDIX B

\section{CCF Correlograms}




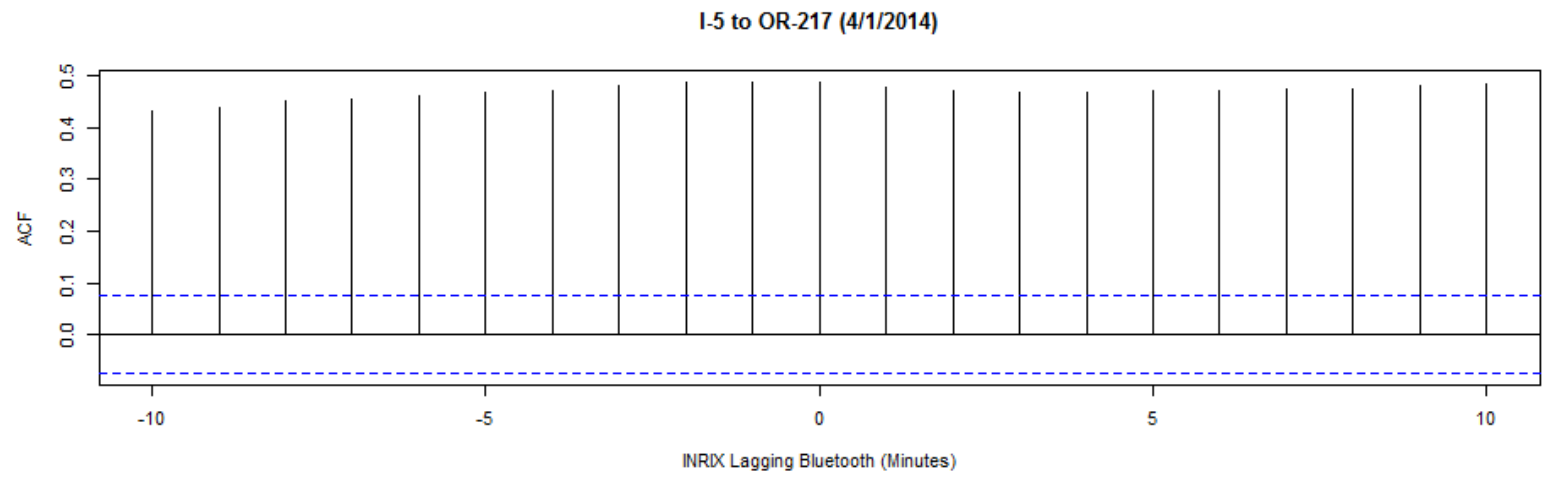

I-5 to OR-217 (4/3/2014)

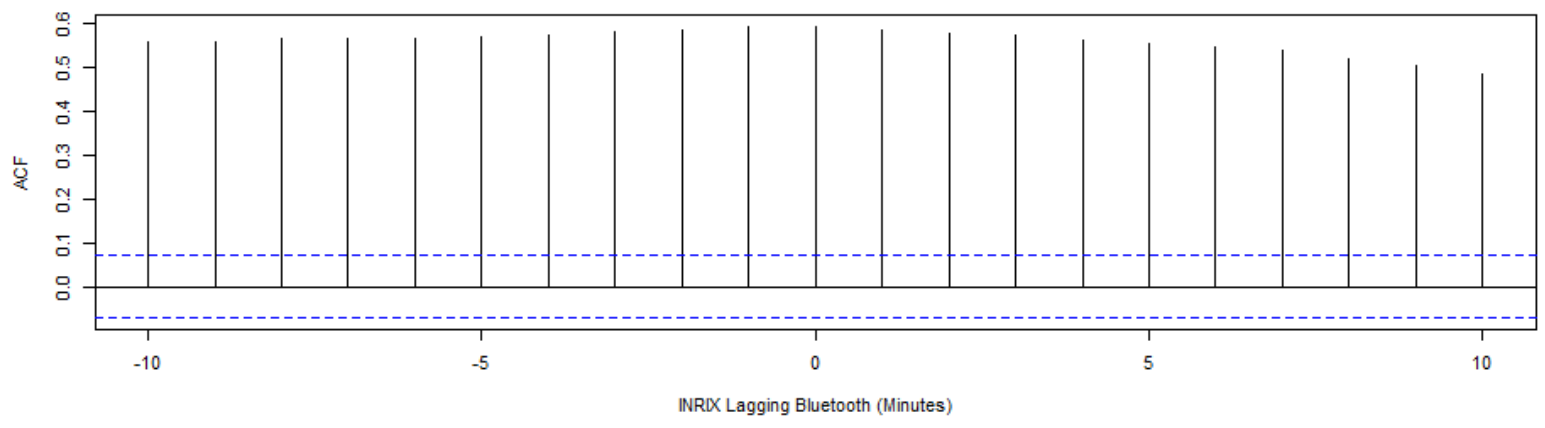

I-5 to OR-217 (4/5/2014)

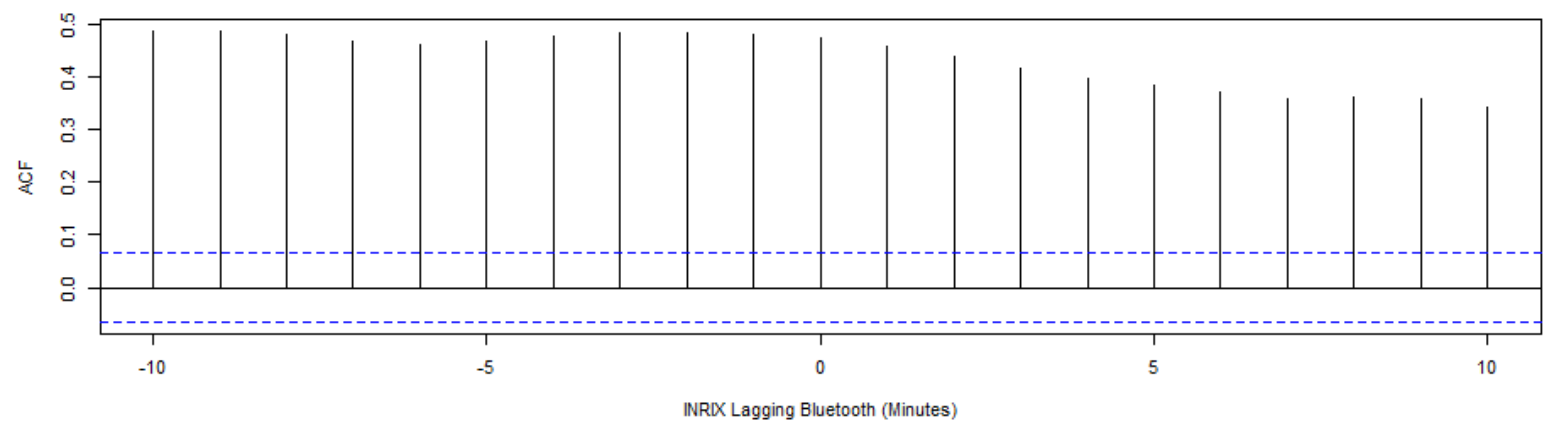

Figure 17: CCF Correlogram for I-5 to OR-217 


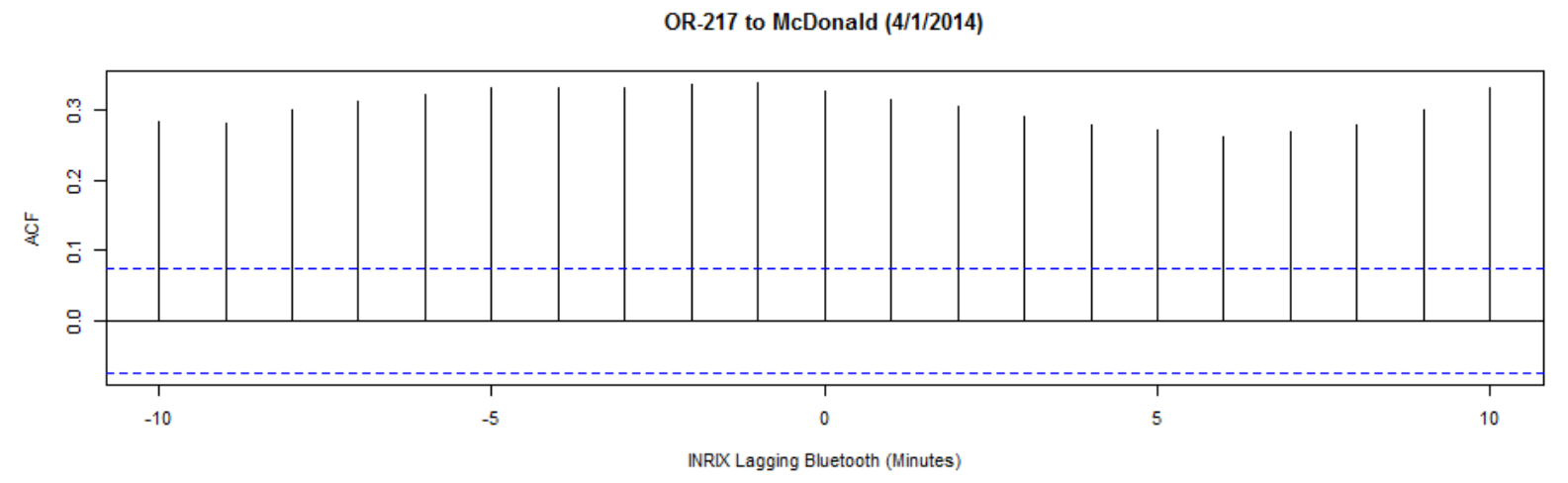

OR-217 to McDonald (4/3/2014)

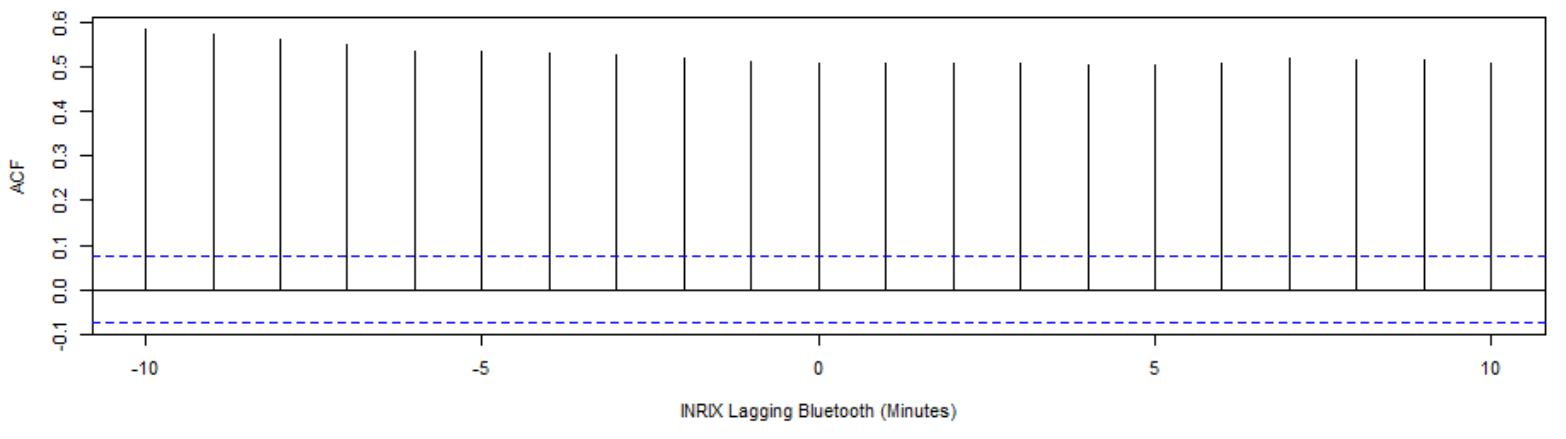

OR-217 to McDonald (4/5/2014)

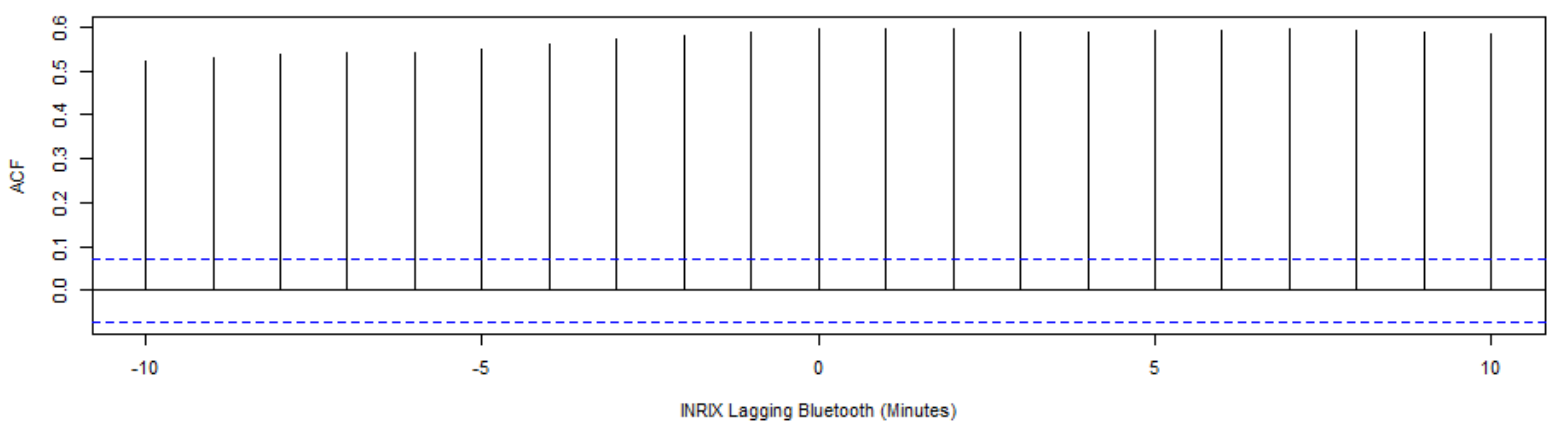

Figure 18: CCF Correlogram for OR-217 to McDonald 


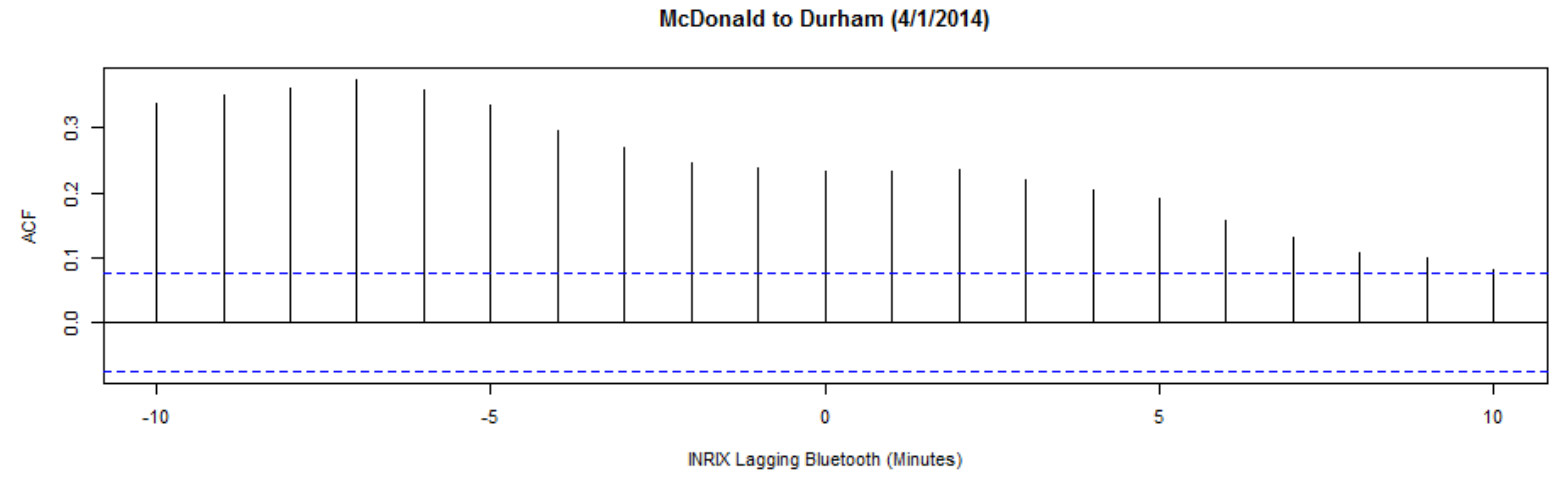

McDonald to Durham (4/3/2014)

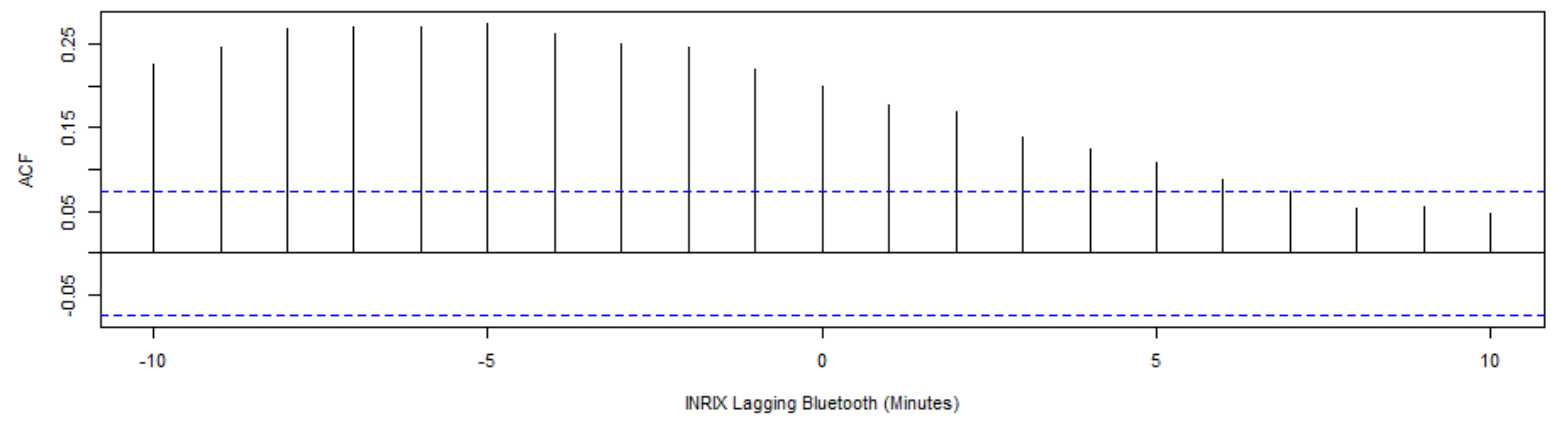

McDonald to Durham (4/5/2014)

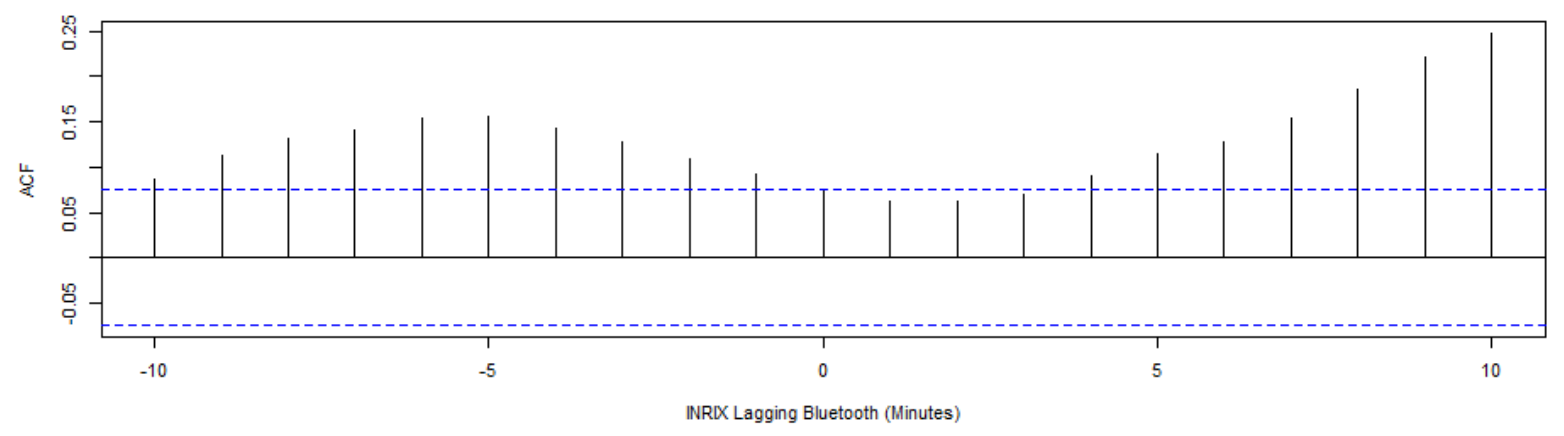

Figure 19: CCF Correlogram for McDonald to Durham 
Durham to McDonald (4/1/2014)

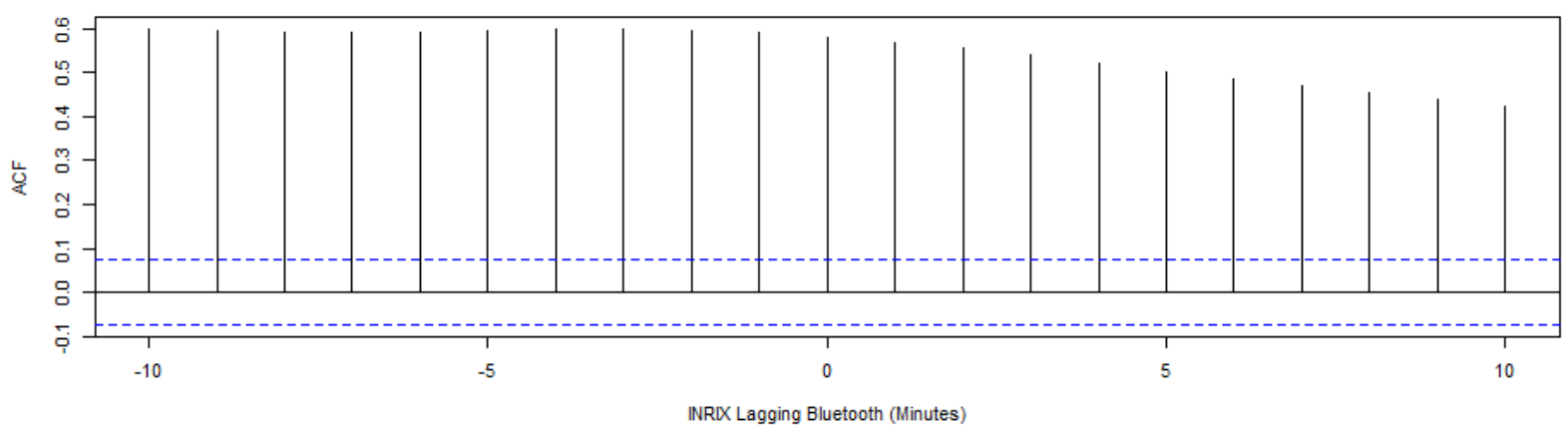

Durham to McDonald (4/3/2014)

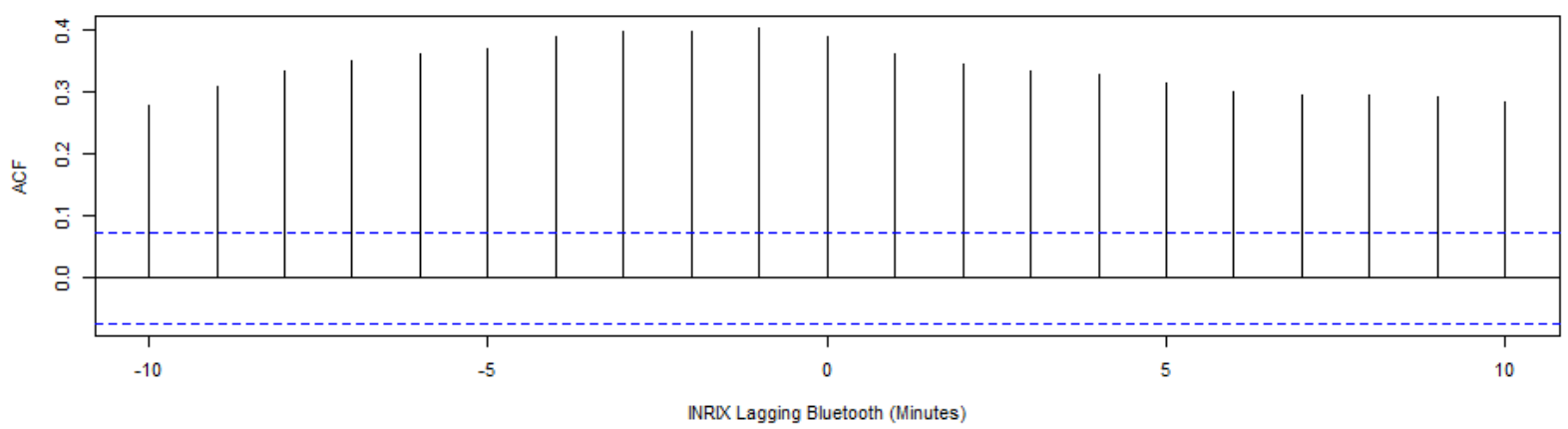

Durham to McDonald (4/5/2014)

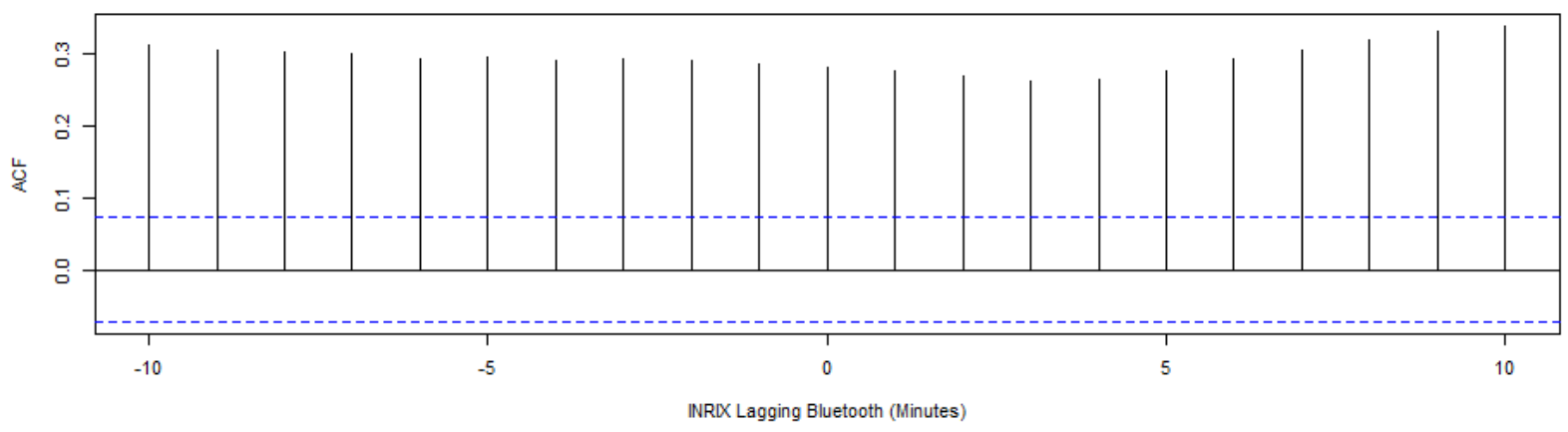

Figure 20: CCF Correlogram for Durham to McDonald 


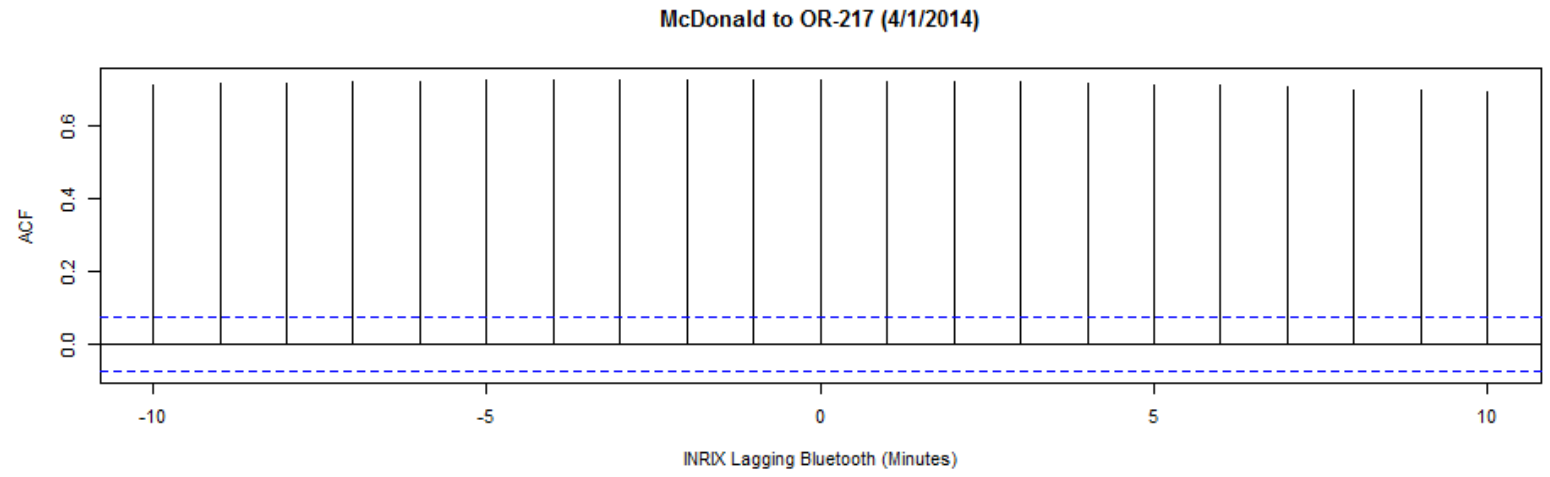

McDonald to OR-217 (4/3/2014)

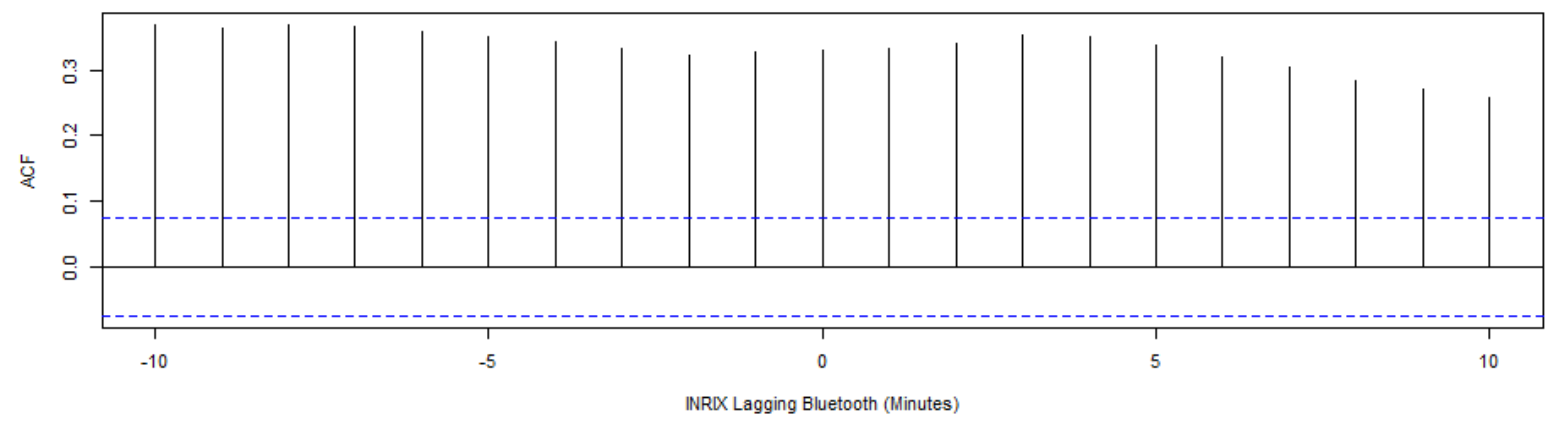

McDonald to OR-217 (4/5/2014)

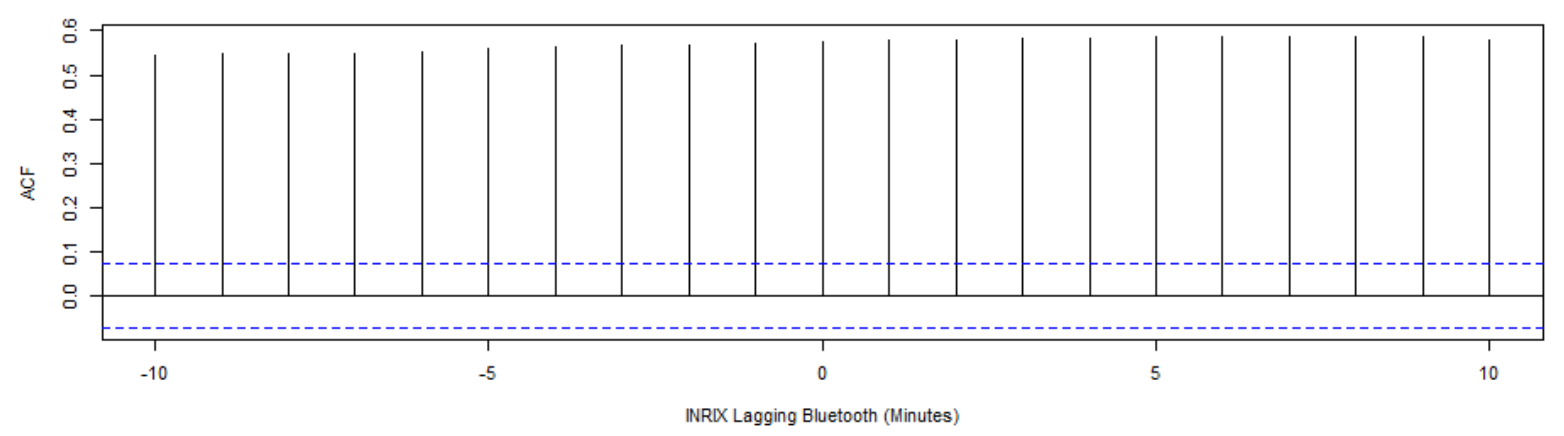

Figure 21: CCF Correlogram for McDonald to OR-217 
OR-217 to I-5 (4/1/2014)

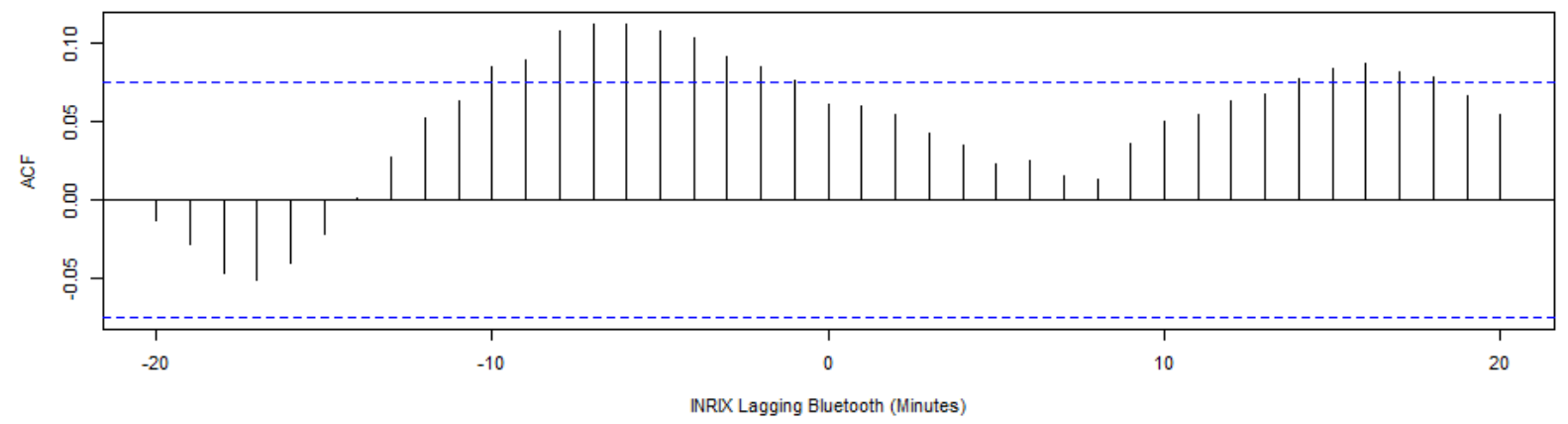

OR-217 to I-5 (4/3/2014)

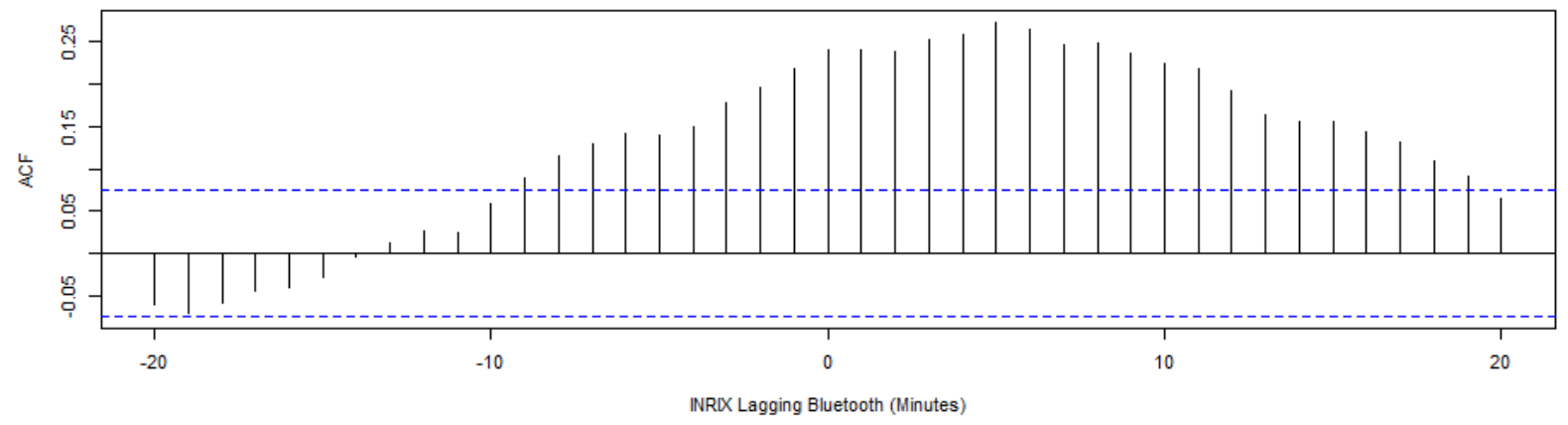

OR-217 to I-5 (4/5/2014)

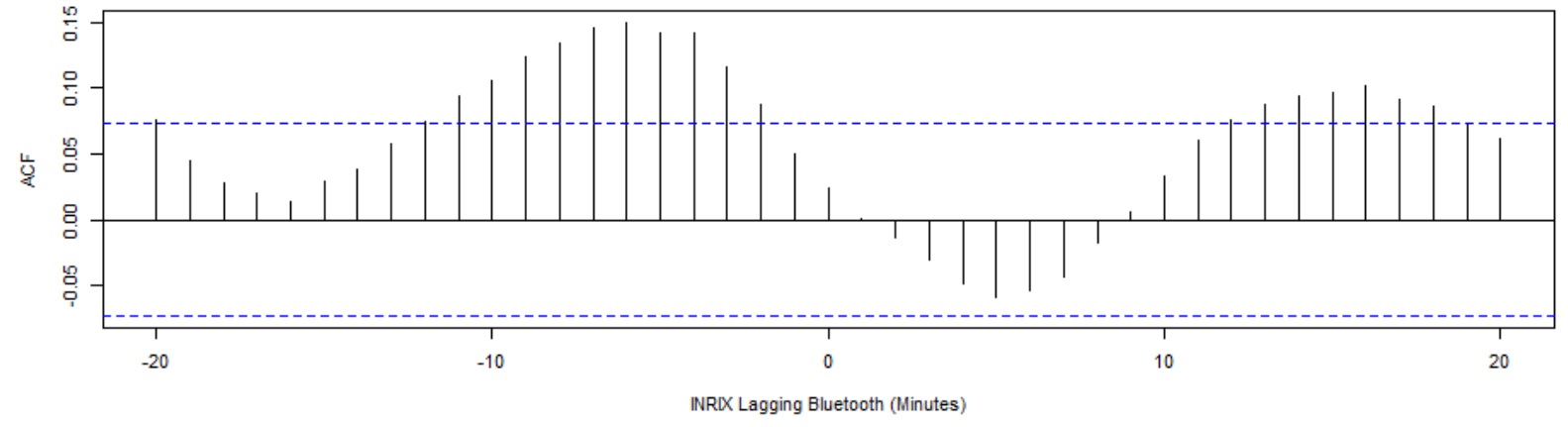

Figure 22: CCF Correlogram for OR-217 to I-5 